\title{
Rate Selection for Wireless Random Access Networks Over Block Fading Channels
}

\author{
Nurullah Karakoç ${ }^{\circledR}$, Student Member, IEEE, and Tolga M. Duman ${ }^{\circledR}$, Fellow, IEEE
}

\begin{abstract}
We study uncoordinated random access over fading channels where each user independently decides whether to send a packet or not to a common receiver at any given time slot. Specifically, we develop an information theoretic formulation to characterize the overall system throughput. We consider two scenarios: classical slotted ALOHA, where no multiuser detection (MUD) capability is available and slotted ALOHA with MUD. In each case, in order to maximize the system throughput, we provide methods to obtain the optimal rates and channel activity probabilities using the user distances to the receiver (or, equivalently, their average signal to noise ratios) assuming a Rayleigh block fading channel. The results demonstrate that the newly proposed optimal rate selection solutions offer significant increase in the expected system throughputs compared to the "same rate to all users" approach commonly used in the literature. In addition to the overall throughput optimization, we also address the issue of fairness among users and propose approaches guaranteeing a minimum amount of individual throughput to each user, and design systems with limited individual outage probabilities for increased energy efficiency and reduced delay.
\end{abstract}

Index Terms-Slotted ALOHA, Rayleigh fading, rate selection, multi-user communications.

\section{INTRODUCTION}

$\mathbf{R}$ ANDOM access schemes offer attractive solutions to the shared receiver (channel) problems due to their distributed and simple nature, and hence, there is a growing interest in them in recent years [3]. As a concrete example, the recently developed long range wide area networking (LoRaWAN [4]) and narrowband IoT (NB-IoT [5]) technologies offer long range, low power IoT connectivity, and in the uplink, they use protocols based on ALOHA [6], [7]. Also, slotted ALOHA is still used as the initial access scheme in satellite communications [8]. With the proliferation of applications requiring random access over wireless links, traditional solutions should be modified by taking into account the wireless channel characteristics including channel variations, thermal noise,

Manuscript received May 12, 2019; revised November 2, 2019; accepted December 17, 2019. Date of publication December 27, 2019; date of current version March 18, 2020. This article is primarily based on Nurullah Karakoç's M.S. Thesis completed at Bilkent University [1], and it was presented in part at the IEEE Global Communications Conference (GLOBECOM), Singapore, December 2017 [2]. The associate editor coordinating the review of this article and approving it for publication was S. Rini. (Corresponding author: Tolga M. Duman.)

Nurullah Karakoç was with the Department of Electrical and Electronics Engineering, Bilkent University, TR-06800 Ankara, Turkey. He is now with the School of Electrical, Computer and Energy Engineering, Arizona State University, Tempe, AZ 85281 USA (e-mail: nkarakoc@asu.edu).

Tolga M. Duman is with the Department of Electrical and Electronics Engineering, Bilkent University, TR-06800 Ankara, Turkey (e-mail: duman@ee.bilkent.edu.tr).

Color versions of one or more of the figures in this article are available online at http://ieeexplore.ieee.org.

Digital Object Identifier 10.1109/TCOMM.2019.2962799 multiuser interference along with the availability of channel state information (CSI), and novel approaches should be devised. It is also imperative to consider the asymmetry of distances among the devices and the common receiver, especially, in long range applications (LoRa [9]), resulting in very different average signal to noise ratios (SNRs).

In classical slotted ALOHA networks [10], [11], single user detection (SUD) is used, i.e., collisions result in loss of all colliding packets. That is, each successfully received packet is the result of a point-to-point transmission without any interference, and the maximum achievable normalized throughput is 0.368 packets/slot [12]. This rate can be increased by about $50 \%$ by considering the capture effect [13], which allows for the recovery of only the strongest signal in a collision. On the other hand, recent works mostly focus on recovering as many packets as possible in the event of collision with multi-user detection (MUD) methods [14]. These works can be classified into those that provide improvements in the medium access control layer where the focus is on the creating new transmission protocols [15], and those that provide improvements in the physical layer.

The works that focus on physical layer improvements mostly approach the problem from an information theoretic perspective, and focus on rate selection. Médard et. al. [16] provide a method to increase the sum-rate of slotted ALOHA over an additive white Gaussian noise (AWGN) channel with the help of superposition coding and rate splitting. Ref. [17] extends this approach to (ergodic) Rayleigh fading channels. Channel-aware ALOHA, where transmitters have CSI knowledge and utilize multiuser diversity, is studied in [18], [19]. A physical layer network coding strategy is applied for random access in [20] and a non-orthogonal multiple access (NOMA) based improvement is proposed in [21]. In [22], the authors propose rate adaptation of the encoding rate according to the channel load to improve the system throughput by allowing MUD and compare the results with information theoretic limits. Throughput maximization with random arrivals in both coordinated and uncoordinated setups with the same rate assignment to all the users is studied in [23], and coding schemes for massive random access are studied in [24]. In addition, in [25], the authors characterize the maximum sum-rate of slotted ALOHA with successive interference cancellation (SIC), where optimum activity probability and encoding rate are obtained considering equal mean received SNRs, and correspondingly, equal rate allocations for different users.

In this paper, we consider many probabilistically active users with different distances to a common receiver. The users 
transmit their packets over a shared wireless channel modeled as slow (non-ergodic) Rayleigh fading, and we assume that the CSI is only known at the receiver. We approach the rate selection problem from an information-theoretic perspective at the physical layer without making any changes at the medium access control layer of the standard slotted ALOHA framework. To preserve the simple nature of ALOHA, no coordination among users is considered, and sophisticated schemes such as rate splitting and superposition coding are avoided.

Our novel contributions are as follows: we characterize the optimal user rates to maximize the system throughput for both single-user detection and MUD cases (modeling each collision as a Gaussian multiple access channel (MAC) with fading), and show that assignment of different rates to users with different distances (i.e., with different average SNRs) provides a significant increase in the expected throughput compared to the commonly used "same rate to all users" approach (see, e.g., [22], [23], [25]), especially, if the users are spatially separated. Rather than employing superposition coding over AWGN [16] or ergodic fading scenarios [17], we adopt a constant rate approach for a given user throughout its transmission over (non-ergodic) block fading channels. We also present possible ways of including additional criteria such as fairness and limited outage probabilities into the optimization framework, and obtain optimal rates and activity probabilities for different scenarios.

The paper is organized as follows. In Section II, we introduce the system model. In Sections III and IV, we present the newly proposed methods for optimal rate and activity probability selection in classical slotted ALOHA and slotted ALOHA with MUD, respectively. In Section V, we discuss several practical issues including implementation of the proposed solutions with minimal feedback, and in Section VI, we provide numerical examples to illustrate our findings and make comparisons with the related approaches in the literature. Finally, we conclude the paper in Section VII.

\section{System ModeL}

We consider a slotted ALOHA system over a communication medium characterized as a wireless channel with pathloss and small scale fading effects. As illustrated in Fig. 1, we assume that there are $n$ users distributed over a ring of inner radius $d_{\min }$ and outer radius $d_{\max }$, and there is a common receiver at the center of the ring. User $i$ is active, i.e., sends a packet, with probability $p_{i}$ where $0 \leq p_{i} \leq 1$, and it has a distance $d_{i}$ to the common receiver (with $d_{\min } \leq d_{i} \leq d_{\max }$ ), where $i=1, \ldots, n$. We assume that the queue of a user is non-empty, i.e., the users always have a packet to send when they are active, since information regarding queue lengths is typically important for scheduling while we only focus on uncoordinated transmissions. We also assume that the receiver knows the identity of randomly active users in a time $\operatorname{slot}^{1}$.

We use a simplified path-loss model to determine the average SNR for each user, however, other channel effects such as shadowing can also be taken into account in a similar manner. The received power $P_{i}$ corresponding to the user $i$ 's

\footnotetext{
${ }^{1}$ We discuss how this can be accomplished in practice in Sec. V.
}

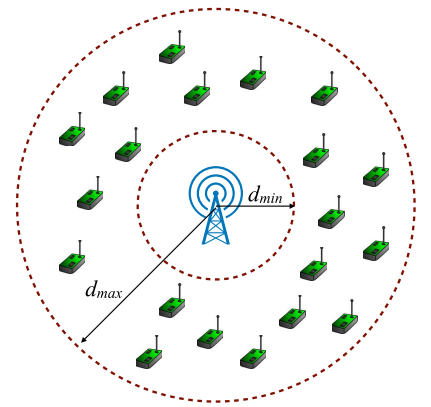

Fig. 1. Users are distributed over a ring of inner radius $d_{\min }$ and outer radius $d_{\max }$

signal is given by

$$
P_{i}=P_{t} \kappa\left(\frac{d_{0}}{d_{i}}\right)^{\gamma} \text { for } i=1,2, \ldots, n,
$$

where $\gamma$ is the path-loss exponent, $P_{t}$ is the transmit power assumed to be the same for all the users, $\kappa$ and $d_{0}$ are constants. To model the small scale fading effects, we consider Rayleigh fading, and assume that the CSI is known at the receiver side only. We also assume that the channel is slowly varying and the channel gain can be modeled as constant over each slot (which is long enough to invoke the relevant random coding arguments).

For simplicity of exposition, we take the channel coefficients as real Rayleigh random variables, which are assumed known at the receiver. Conditioned on the instantaneous channel gain $h_{i}$, the point-to-point capacity over a fading channel with AWGN is

$$
C\left(P_{i} h_{i}^{2}\right)=\frac{1}{2} \log _{2}\left(1+\frac{P_{i} h_{i}^{2}}{N}\right) \text { bits/channel use }
$$

where $C(x)=\frac{1}{2} \log _{2}\left(1+\frac{x}{N}\right)$, and $N$ denotes the additive noise power. Hence, with a channel gain of $h_{i}$, a rate of $R_{i}<C\left(P_{i} h_{i}^{2}\right)$ can be supported reliably.

For a Gaussian MAC with two users, conditioned on the channel gains $h_{i}$ and $h_{j}$, the capacity region is

$$
\begin{aligned}
R_{i} & <C\left(P_{i} h_{i}^{2}\right) \\
R_{j} & <C\left(P_{j} h_{j}^{2}\right) \\
R_{i}+R_{j} & <C\left(P_{i} h_{i}^{2}+P_{j} h_{j}^{2}\right)
\end{aligned}
$$

where $\left(R_{i}, R_{j}\right)$ is the users' rate pair [26]. Fig. 2 illustrates two different examples of the capacity region for two different sets of channel realizations. All rate pairs that can be supported reliably are in the shaded pentagonal regions represented by (3). A selected rate pair $\left(R_{i}^{*}, R_{j}^{*}\right)$ (marked in the figures) can be supported for the example on the left hand side, while it is outside the capacity region for the one on the right hand side. Namely, for the latter case, the users experience outage due to channel fading.

\section{Optimal Rates And Activity Probabilities IN SLOTTED ALOHA}

\section{A. Optimal Rate Selection}

In classical slotted ALOHA (without MUD at the receiver), collisions result in a loss of all the colliding packets. Therefore, 


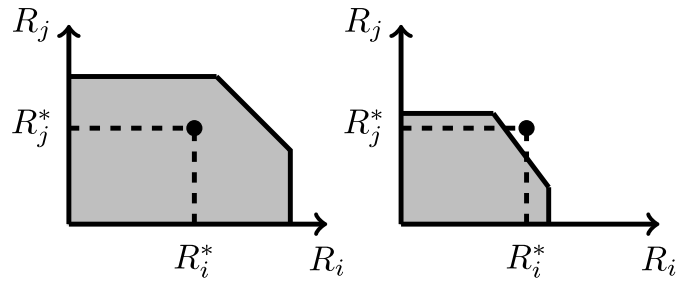

Fig. 2. Two-user Gaussian MAC capacity regions for different fading realizations.

achievable rates are identified with the single-user capacity in (2). In order to achieve a successful transmission in a given time slot, there should only be one packet in that particular slot, and the active user's rate should be supported by the specific channel realization. Denoting the encoding rate of a user with distance $d_{i}$ to the common receiver by $R\left(d_{i}\right)$, the throughput of the user $i$ in a given slot, conditioned on the event that only that user transmits in the particular slot is given by

$$
T_{i}= \begin{cases}R\left(d_{i}\right), & \text { if } R\left(d_{i}\right)<C\left(P_{i} h_{i}^{2}\right) \\ 0, & \text { otherwise }\end{cases}
$$

where $h_{i}$ denotes the channel gain. Denoting the expected throughput in a slot by $T$ and using the law of total expectation, we obtain

$$
\begin{aligned}
T & =\sum_{i=1}^{n} \mathbb{E}_{h_{i}}\left[T_{i}\right] \operatorname{Pr}(\text { only } i \text { transmits }) \\
& =\sum_{i=1}^{n} R\left(d_{i}\right) \operatorname{Pr}\left(R\left(d_{i}\right)<C\left(P_{i} h_{i}^{2}\right)\right) \operatorname{Pr}(\text { only } i \text { transmits })
\end{aligned}
$$

where $\operatorname{Pr}(\cdot)$ denotes probability, and the expectation $\mathbb{E}_{h_{i}[\cdot] \text { is }}$ taken over the random channel gains. Since transmissions are modeled as independent Bernoulli trials,

$$
\operatorname{Pr}(\text { only } i \text { transmits })=p_{i} \prod_{\substack{j=1 \\ j \neq i}}^{n}\left(1-p_{j}\right) .
$$

Noting that $h_{i}^{2}$ is an exponential random variable with mean 1 , the probability of no outage is given by

$$
\begin{aligned}
\operatorname{Pr}\left(R\left(d_{i}\right)<C\left(P_{i} h_{i}^{2}\right)\right) & =\operatorname{Pr}\left(R\left(d_{i}\right)<\frac{1}{2} \log _{2}\left(1+\frac{P_{i} h_{i}^{2}}{N}\right)\right) \\
& =e^{-\left(2^{2 R\left(d_{i}\right)}-1\right) \frac{N d_{i}^{\gamma}}{P_{0}}}
\end{aligned}
$$

with $P_{0}=P_{t} \kappa d_{0}^{\gamma}$. By combining (5)-(7), the expected throughput optimization problem becomes

$$
\max _{R\left(d_{i}\right)} \sum_{i=1}^{n} R\left(d_{i}\right) e^{-\left(2^{2 R\left(d_{i}\right)}-1\right) \frac{N d_{i}^{\gamma}}{P_{0}}} p_{i} \prod_{\substack{j=1 \\ j \neq i}}^{n}\left(1-p_{j}\right) .
$$

The optimal rate $R^{*}\left(d_{i}\right)$ of user $i$ can then be characterized as a function of its distance $d_{i}$. By direct differentiation, for optimality, we obtain

$p_{i} \prod_{\substack{j=1 \\ j \neq i}}^{n}\left(1-p_{j}\right) e^{-\left(2^{2 R\left(d_{i}\right)}-1\right) \alpha_{i}}\left[1-2^{2 R\left(d_{i}\right)} \alpha_{i} 2 R\left(d_{i}\right) \ln 2\right]=0$ which results in

$$
1-2^{2 R^{*}\left(d_{i}\right)} \alpha_{i} 2 R^{*}\left(d_{i}\right) \ln 2=0
$$

with $\alpha_{i}=\frac{N d_{i}^{\gamma}}{P_{0}}$. That is, the optimal rate is given by

$$
R^{*}\left(d_{i}\right)=\frac{W\left(\frac{P_{0}}{N d_{i}^{\gamma}}\right)}{\ln (4)}
$$

where $W(\cdot)$ is the Lambert-W function ${ }^{2}$, i.e., the inverse of $f(z)=z e^{z}$.

Writing the system throughput in (8) as a summation of the individual throughputs $T^{(i)}$ 's where $T^{(i)}=$ $R\left(d_{i}\right) e^{-\left(2^{2 R\left(d_{i}\right)}-1\right) \alpha_{i}} p_{i} \prod_{\substack{j=1 \\ j \neq i}}^{n}\left(1-p_{j}\right)$, and noting that $R\left(d_{i}\right)$ values have no effect on $T^{(j)}$ for $j \neq i$, we can write the optimal system throughput as $T^{*}=\sum_{i=1}^{n} T_{o p t}^{(i)}$ with $T_{o p t}^{(i)}=$ $\max _{R\left(d_{i}\right)}\left\{T^{(i)}\right\}$. The first order partial derivative of $T^{(i)}$ with respect to $R\left(d_{i}\right)$ is given by

$$
\left\{\begin{array}{l}
\frac{\partial T^{(i)}}{\partial R\left(d_{i}\right)}>0, \text { if } 0 \leq R\left(d_{i}\right)<R^{*}\left(d_{i}\right)=\frac{W\left(\frac{P_{0}}{N d_{i}^{\gamma}}\right)}{\ln (4)}, \\
\frac{\partial T^{(i)}}{\partial R\left(d_{i}\right)} \leq 0, \text { otherwise, }
\end{array}\right.
$$

which indicates that the objective function is increasing before the optimal point, and non-increasing after, proving that the result in (10) is in fact a globally optimal solution for the overall throughput maximization problem.

\section{B. System Design With Fairness}

Optimal rate function in (10) states that the closer users to the destination have higher optimal rates, and hence for the case of identical activity probabilities, i.e., $p_{1}=p_{2}=\ldots=$ $p_{n}$, they enjoy higher individual throughputs compared to the far away users. We now propose a method that adjusts the activity probabilities and guarantees a minimum amount of individual throughput to each user by allowing the far away users to send their packets more frequently.

For simplicity of calculations, the users are divided into $k$ groups in terms of their distances, and we assume that the number of active users in each group in a given slot is modeled as a Poisson random variable with parameter $\lambda_{j}=n_{j} p_{j}$ where $j=1,2, \ldots, k$ denotes the group index, $d_{j}$ denotes the distance of group $j$ users to the receiver, and $n_{j}$ and $p_{j}$ are number of users and the activity probability of the users in group $j$, respectively. $\lambda_{j}$ is defined as the $j^{\text {th }}$ group's load, and $\sum_{j=1}^{k} \lambda_{j}$ is the channel load.

The probability that there is only one active user in the group $j$ and there are no other active users for a given slot is $\lambda_{j} \exp \left(-\sum_{i=1}^{k} \lambda_{i}\right)$. Then, by using (8) and (10), the optimization problem can be written as

$$
\begin{aligned}
\max _{\lambda_{1}, \lambda_{2}, \ldots, \lambda_{k}}\left(e^{-\sum_{i=1}^{k} \lambda_{i}}\right) \sum_{j=1}^{k} \lambda_{j} r_{j} \\
\text { s.t. }\left(e^{-\sum_{i=1}^{k} \lambda_{i}}\right) \lambda_{j} r_{j} \geq K, \quad \lambda_{j} \geq 0, j=1,2, \ldots, k,
\end{aligned}
$$

\footnotetext{
${ }^{2}$ We note that, this result is derived in [27] as well.
} 
where $K$ is the minimum throughput required for each group, and $r_{j}$ is the effective rate of group $j$ users given by

$$
r_{j}=R^{*}\left(d_{j}\right) e^{-\left(2^{2 R^{*}\left(d_{j}\right)}-1\right) \frac{N d_{j}^{\gamma}}{P_{0}}} .
$$

Notice that $r_{j}$ is independent of the group load $\lambda_{j}$, and it can be taken as a constant in the optimization problem. The Lagrangian $L$ is formed as

$L=\sum_{j=1}^{k}-\left(e^{-\sum_{i=1}^{k} \lambda_{i}}\right) \lambda_{j} r_{j}-\mu_{j} \lambda_{j}-\nu_{j}\left(\lambda_{j} r_{j}\left(e^{-\sum_{i=1}^{k} \lambda_{i}}\right)-K\right)$,

where $\mu_{1}, \mu_{2}, \ldots, \mu_{k} \geq 0$, and $\nu_{1}, \nu_{2}, \ldots, \nu_{k} \geq 0$. For the optimal solution, we have

$\nu_{j}\left(\lambda_{j} r_{j}\left(e^{-\sum_{i=1}^{k} \lambda_{i}}\right)-K\right)=0, \quad \frac{\partial L}{\partial \lambda_{j}}=0, \quad \mu_{j} \lambda_{j}=0, \forall j$.

Then, we obtain $\frac{\partial L}{\partial \lambda_{j}}=\left(r_{j}+\nu_{j} r_{j}-\sum_{i=1}^{k} \lambda_{i} r_{i}-\right.$ $\left.\sum_{i=1}^{k} \lambda_{i} r_{i} \nu_{i}\right) e^{-\sum_{i=1}^{k} \lambda_{i}}=0, \forall j$, and

$$
r_{j}+\nu_{j} r_{j}=\sum_{i=1}^{k} \lambda_{i} r_{i}+\sum_{i=1}^{k} \lambda_{i} r_{i} \nu_{i}, \quad j=1,2, \ldots, k .
$$

Let us denote the right hand side by $\tau$, (i.e., $\tau=\sum_{i=1}^{k} \lambda_{i} r_{i}+$ $\left.\sum_{i=1}^{k} \lambda_{i} r_{i} \nu_{i}\right)$. If we multiply the $j^{\text {th }}$ equation with $\lambda_{j}$ for all $j=1,2, \ldots, k$, and then add them up, we obtain $\tau=\sum_{j=1}^{k} \lambda_{j}\left(r_{j}+\nu_{j} r_{j}\right)=\sum_{j=1}^{k} \lambda_{j} \tau$, which implies that $\sum_{j=1}^{k} \lambda_{j}=1$ for optimality.

Without loss of generality, assume that $r_{1}>r_{2}, \ldots, r_{k}$ (i.e., the first group is the closest one to the receiver). From (16), $r_{1}+\nu_{1} r_{1}=r_{2}+\nu_{2} r_{2}=\ldots=r_{k}+\nu_{k} r_{k}$, and since $r_{1}>r_{2}, \ldots, r_{k}$, then $\nu_{j}>0$ for $j=2, \ldots, k$. Using (15), we then obtain $\lambda_{j} r_{j}\left(e^{-\sum_{i=1}^{k} \lambda_{i}}\right)=K$ for $j=2, \ldots, k$. Hence, the optimal group loads are found as

$$
\lambda_{1}=1-\sum_{j=2}^{k} \frac{K e}{r_{j}} \text { and } \lambda_{j}=\frac{K e}{r_{j}}, \quad j=2,3, \ldots, k,
$$

and the optimal activity probabilities of the users in group $j$ are calculated as $p_{j}=\lambda_{j} / n_{j}$ for $j=1,2, \ldots, k{ }^{3}$

We observe that independent of the value of $K$, the optimal channel load is the same all the time, i.e., $\lambda_{1}+\ldots+\lambda_{k}=$ 1. In other words, all the groups have just enough load for guaranteeing a throughput of $K$, and then the remaining load is assigned to the group with the highest effective rate $r_{j}$, namely, the closest one to the receiver (i.e., the one with the highest average SNR).

We can also consider a fully fair system in which all the groups have equal throughputs. Namely, we can solve

$$
\max _{K, \lambda_{1}, \lambda_{2}, . ., \lambda_{k} \geq 0} K \text { s.t. }\left(e^{-\sum_{i=1}^{k} \lambda_{i}}\right) \lambda_{j} r_{j}=K, j=1,2, . ., k \text {. }
$$

Following similar steps as in the previous optimization procedure, the optimal threshold $K^{*}$ and the optimal load $\lambda_{j}^{*}$

\footnotetext{
${ }^{3}$ Global optimality of the solution is verified in Appendix A by using the fact that objective function and constraints in (12) are log-concave functions of $\lambda_{j}$ 's.
}

for each group can be found as

$$
K^{*}=\frac{1}{e}\left(\sum_{i=1}^{k} \frac{1}{r_{i}}\right)^{-1}, \quad \lambda_{j}^{*}=\frac{1}{r_{j}}\left(\sum_{i=1}^{k} \frac{1}{r_{i}}\right)^{-1},
$$

with $j=1,2, \ldots, k$. These results imply that a fully fair system can be achieved by only using users' effective rate (i.e., their average SNR) information.

\section{Limiting Individual Outage Probabilities}

Optimal rates in (10) may result large outage probabilities for some of the users. Since outage of each packet leads to a retransmission, it may not be energy efficient or delay efficient to use these rates for some of the users. To handle this issue, we modify the optimization problem in (8) by incorporating the individual outage probability constraints. Therefore, we solve

$$
\begin{aligned}
& \max _{R\left(d_{i}\right)} \sum_{i=1}^{n} R\left(d_{i}\right) e^{-\left(2^{2 R\left(d_{i}\right)}-1\right) \alpha_{i}} p_{i} \prod_{\substack{j=1 \\
j \neq i}}^{n}\left(1-p_{j}\right) \\
& \text { s.t. } \operatorname{Pr}\left(R\left(d_{i}\right)>C\left(P_{i} h_{i}^{2}\right)\right) \leq \beta_{i}, \forall i \in\{1,2, \ldots, n\}
\end{aligned}
$$

where $\beta_{i}$ is the maximum allowed individual outage probability for user $i$. Since each transmission is point-to-point, i.e., independent of each other, and activity probabilities are constant, we can simplify (19) as

$$
\begin{aligned}
& \max _{R\left(d_{i}\right)} R\left(d_{i}\right) e^{-\left(2^{2 R\left(d_{i}\right)}-1\right) \alpha_{i}} \\
& \text { s.t. } 1-e^{-\left(2^{2 R\left(d_{i}\right)}-1\right) \alpha_{i}} \leq \beta_{i},
\end{aligned}
$$

where the outage probability characterized in (7) is employed.

To solve the problem in (20), we form the Lagrangian

$$
L=-R\left(d_{i}\right) e^{-\left(2^{2 R\left(d_{i}\right)}-1\right) \alpha_{i}}+\mu\left(1-e^{-\left(2^{2 R\left(d_{i}\right)}-1\right) \alpha_{i}}-\beta_{i}\right),
$$

where $\mu$ is a Lagrange multiplier. From the Karush-KuhnTucker (KKT) conditions, we obtain

$$
\begin{aligned}
0= & \left(e^{-\left(2^{2 R\left(d_{i}\right)}-1\right) \alpha_{i}}\left(1-2^{2 R\left(d_{i}\right)} \alpha_{i} 2 R\left(d_{i}\right) \ln 2\right)\right) \\
& +\mu e^{-\left(2^{2 R\left(d_{i}\right)}-1\right) \alpha_{i}} 2^{2 R\left(d_{i}\right)} \alpha_{i} 2 \ln 2 \\
=1- & \left(R\left(d_{i}\right)+\mu\right) 2^{2 R\left(d_{i}\right)} \alpha_{i} 2 \ln 2
\end{aligned}
$$

and

$$
\mu\left(1-e^{-\left(2^{2 R\left(d_{i}\right)}-1\right) \alpha_{i}}-\beta_{i}\right)=0, \quad \mu \geq 0 .
$$

By combining (21) and (22), the resulting optimal rate of user $i$ is given by

$$
R^{*}\left(d_{i}\right)=\min \left(\frac{W\left(\frac{P_{0}}{N d_{i}^{\gamma}}\right)}{\ln (4)}, \frac{\ln \left(1-\frac{\ln \left(1-\beta_{i}\right)}{\alpha_{i}}\right)}{\ln (4)}\right),
$$

which is indeed globally optimal since the objective function is increasing in $R\left(d_{i}\right)$ below and either non-increasing or not feasible (i.e., constraint is violated) above the optimum point. We also note that limitation of the outage probabilities and fairness can also be combined by modifying the effective rates in (13) with the values in (23). 


\section{Slotted Aloha With Multi-User Detection}

\section{A. Optimal Rate Selection}

We now extend our approach in the previous section to systems with a (common) receiver with MUD capabilities. We model each collision as a Gaussian MAC with fading, and denote the rate of user $i$ by $R_{i}$ where $i=1,2, \ldots, n$. We note that the optimal rates $R_{i}$ 's are functions of distances of all the users, which are assumed as known. ${ }^{4}$ Noting that there exist implementable (practical) coding schemes with two users over a MAC [28], [29], we assume that the MUD capability is limited to the collisions of two packets and declare the packets as lost if more than two collide. For the expected throughput formulation, we modify (5) with the addition of decodable two-user collisions. Therefore, in the formulation, there is a summation of $n$ terms for single level decoding (when there is only one user active in a slot), and there are $\left(\begin{array}{l}n \\ 2\end{array}\right)$ additional terms for two-level decoding (which means that the decoder may be successful when at most two packets collide). For each of these $\left(\begin{array}{l}n \\ 2\end{array}\right)$ terms, there are three inequalities specified in (3) for two-user Gaussian MAC capacity determining whether the decoding process is successful or there is a decoder failure.

As a side note, we note that for $m$-level decoding, there are $\left(\begin{array}{c}n \\ m\end{array}\right)$ additional terms and for each of them $2^{m}-1$ additional inequalities are needed compared to the $(m-1)$-level decoding. Hence, while the formulation here can be easily extended, the calculations needed for the optimization will be very high. Also, we exclude the cases where one of the two users can be decoded by treating the other as noise (i.e., we assume the users are either both decodable or both non-decodable in a collision $)^{5}$.

The expected throughput in (5) can then be written as

$$
\begin{aligned}
T= & \sum_{i=1}^{n} R_{i} \operatorname{Pr}\left(R_{i}<C\left(P_{i} h_{i}^{2}\right)\right) p_{i} \prod_{\substack{j=1 \\
j \neq i}}^{n}\left(1-p_{j}\right) \\
& +\sum_{i=1}^{n-1} p_{i} \sum_{j=i+1}^{n} p_{j} \prod_{\substack{k=1 \\
k \neq i, j}}^{n}\left(1-p_{k}\right)\left(R_{i}+R_{j}\right) A\left(R_{i}, R_{j}\right),
\end{aligned}
$$

where $A\left(R_{i}, R_{j}\right)$ denotes the two-user non-outage probability. We define $\eta_{i}=P_{i} h_{i}^{2} / N$ where $f_{\eta_{i}}(x)$ denotes the probability density function (p.d.f.) of $\eta_{i}$ with $h_{i}^{2}$ and $h_{j}^{2}$ being independent and identically distributed (i.i.d.) exponential random variables with mean 1 . We write

$$
\begin{array}{r}
A\left(R_{i}, R_{j}\right)=\operatorname{Pr}\left(R_{i}<C\left(P_{i} h_{i}^{2}\right), R_{j}<C\left(P_{j} h_{j}^{2}\right),\right. \\
\left.R_{i}+R_{j}<C\left(P_{i} h_{i}^{2}+P_{j} h_{j}^{2}\right)\right) \\
=\operatorname{Pr}\left(\eta_{i} \geq 2^{2 R_{i}}-1, \eta_{j} \geq 2^{2 R_{j}}-1,\right. \\
\left.\eta_{i}+\eta_{j} \geq 2^{2\left(R_{i}+R_{j}\right)}-1\right)
\end{array}
$$

\footnotetext{
${ }^{4}$ Note that, in the SUD case considered in the previous section, the optimal rate of a particular user is only a function of the distance of that user to the receiver.

${ }^{5}$ For analytical tractability, we employ this assumption throughout the paper, however, we also evaluate the performance of the proposed method for decoding with capture (see Sec. VI), where we observe that the proposed method performs well in low activity probabilities. Due to the fact that ALOHA networks are used in applications with low activity probabilities in general, this assumption appears practical.
}

$$
\begin{gathered}
=\int_{2^{2 R_{i}}-1}^{2^{2 R_{j}}\left(2^{2 R_{i}}-1\right)} f_{\eta_{i}}(x) \int_{2^{2\left(R_{i}+R_{j}\right)}-1-x}^{\infty} f_{\eta_{j}}(y) \mathrm{d} y \mathrm{~d} x \\
\quad+\int_{2^{2 R_{j}}\left(2^{2 R_{i}}-1\right)}^{\infty} f_{\eta_{i}}(x) \int_{2^{2 R_{j}-1}}^{\infty} f_{\eta_{j}}(y) \mathrm{d} y \mathrm{~d} x .
\end{gathered}
$$

For $\alpha_{i} \neq \alpha_{j}$, this expression can be evaluated in closed form as

$$
\begin{aligned}
A\left(R_{i}, R_{j}\right)= & \int_{2^{2 R_{i}}-1}^{2^{2 R_{j}}\left(2^{2 R_{i}}-1\right)} \alpha_{i} e^{-\alpha_{i} x} e^{-\alpha_{j}\left(2^{2\left(R_{i}+R_{j}\right)}-1-x\right)} \mathrm{d} x \\
= & \frac{\alpha_{i}}{\alpha_{j}-\alpha_{i}} e^{-\alpha_{j}\left(2^{2\left(R_{i}+R_{j}\right)}-1\right)} e^{\left(\alpha_{j}-\alpha_{i}\right)\left(2^{\left.2 R_{i}-1\right)\left(2^{2 R_{j}}-1\right)}\right.} \\
& +e^{-\alpha_{j}\left(2^{2 R_{j}}-1\right)} e^{-\alpha_{i}\left(2^{2 R_{i}}-1\right) 2^{2 R_{j}}} .
\end{aligned}
$$

For the case with $\alpha_{i}=\alpha_{j}$, the two-user non-outage probability can be computed as

$$
\begin{aligned}
A\left(R_{i}, R_{j}\right)= & \int_{2^{2 R_{i}}-1}^{2^{2 R_{j}\left(2^{2 R_{i}}-1\right)}} \alpha_{i} e^{-\alpha_{i}\left(2^{2\left(R_{i}+R_{j}\right)}-1\right)} \mathrm{d} x \\
& \quad+e^{-\alpha_{i}\left(2^{2\left(R_{i}+R_{j}\right)}-1\right)} \\
= & \left(1+\alpha_{i}\left(2^{2 R_{i}}-1\right)\left(2^{2 R_{j}}-1\right)\right) \\
& \quad \times \exp \left(-\alpha_{i}\left(2^{2\left(R_{i}+R_{j}\right)}-1\right)\right) .
\end{aligned}
$$

By combining (7), (24), (25) and (26), the optimization problem for optimal rate selection becomes

$$
\begin{aligned}
& \max _{R_{1}, R_{2}, \ldots, R_{n}} \sum_{i=1}^{n} p_{i} \prod_{\substack{j=1 \\
j \neq i}}^{n}\left(1-p_{j}\right) R_{i} \exp \left(-\alpha_{i}\left(2^{2 R_{i}}-1\right)\right) \\
& +\sum_{i=1}^{n-1} p_{i} \sum_{j=i+1}^{n} p_{j} \prod_{\substack{k=1 \\
k \neq i, j}}^{n}\left(1-p_{k}\right)\left(R_{i}+R_{j}\right) A\left(R_{i}, R_{j}\right) .
\end{aligned}
$$

Using this formulation, given the activity probabilities $p_{i}$ 's and the user distances $d_{i}$ 's, the optimal rates $R_{i}^{*}$ 's can be found via numerical tools such as gradient descent algorithms or interior-point methods ${ }^{6}$. We also note that some optimal rates can be zero as the sole objective is the overall throughput maximization. Convergence of the solution to a globally optimal point is discussed next.

Obtaining a Globally Optimal Solution: The problem in (27) is non-convex, hence we cannot guarantee global optimality of any locally optimal solution. In an effort to obtain a globally optimal solution, we modify the original problem and obtain a relaxed version by using an upper bound on the twouser MAC capacity. Along with some additional constraints, we can guarantee that the solution of the modified problem converges to a globally optimal point. We then verify via extensive numerical examples that the results obtained in most cases are (very close to) globally optimal solution of the original problem. Specifically, we use an upper bound on the MAC capacity region defined by $R_{i}+R_{j}<C\left(P_{i} h_{i}^{2}+P_{j} h_{j}^{2}\right)$, i.e., we simply omit the maximum achievable individual rate bounds and only use a sum-rate bound. By using this upper

\footnotetext{
${ }^{6}$ Details of these techniques are not provided in this paper, and can be found in [30, Chp. 9.3 and 11].
} 
bound, for the case of $\alpha_{i} \neq \alpha_{j}$, an upper bound to the nonoutage probability becomes

$$
\begin{aligned}
\bar{A}( & \left.R_{i}, R_{j}\right) \\
= & \operatorname{Pr}\left(R_{i}+R_{j}<C\left(P_{i} h_{i}^{2}+P_{j} h_{j}^{2}\right)\right) \\
= & \operatorname{Pr}\left(\eta_{i}+\eta_{j} \geq 2^{2\left(R_{i}+R_{j}\right)}-1\right) \\
= & \int_{0}^{2^{2\left(R_{i}+R_{j}\right)}-1} f_{\eta_{i}}(x) \int_{2^{2\left(R_{i}+R_{j}\right)}-1-x}^{\infty} f_{\eta_{j}}(y) \mathrm{d} y \mathrm{~d} x \\
& \quad+\int_{2^{2\left(R_{i}+R_{j}\right)}-1}^{\infty} f_{\eta_{i}}(x) \mathrm{d} x \\
= & \int_{0}^{2^{2\left(R_{i}+R_{j}\right)}-1} \alpha_{i} e^{-\alpha_{i} x} e^{-\alpha_{j}\left(2^{2\left(R_{i}+R_{j}\right)}-1-x\right)} \mathrm{d} x \\
& +e^{-\alpha_{i}\left(2^{2\left(R_{i}+R_{j}\right)}-1\right)},
\end{aligned}
$$

which can be evaluated as

$$
\bar{A}\left(R_{i}, R_{j}\right)=\frac{\alpha_{j} e^{-\alpha_{i}\left(2^{2\left(R_{i}+R_{j}\right)}-1\right)}-\alpha_{i} e^{-\alpha_{j}\left(2^{2\left(R_{i}+R_{j}\right)}-1\right)}}{\alpha_{j}-\alpha_{i}} .
$$

Similarly, for the case of $\alpha_{i}=\alpha_{j}$,

$$
\begin{aligned}
\bar{A}\left(R_{i}, R_{j}\right)= & \int_{0}^{2^{2\left(R_{i}+R_{j}\right)}-1} \alpha_{i} e^{-\alpha_{i}\left(2^{2\left(R_{i}+R_{j}\right)}-1\right)} \mathrm{d} x \\
& +e^{-\alpha_{i}\left(2^{2\left(R_{i}+R_{j}\right)}-1\right)} \\
= & \left(\alpha_{i}\left(2^{2\left(R_{i}+R_{j}\right)}-1\right)+1\right) \exp \left(-\alpha_{i}\left(2^{2\left(R_{i}+R_{j}\right)}-1\right)\right) .
\end{aligned}
$$

We modify the throughput expression in (24) by substituting $A\left(R_{i}, R_{j}\right)$ with the upper bound $\bar{A}\left(R_{i}, R_{j}\right)$ as

$$
\begin{aligned}
\widetilde{T}_{M U D}= & \sum_{i=1}^{n} R_{i} e^{-\alpha_{i}\left(2^{2 R_{i}}-1\right)} p_{i} \prod_{\substack{j=1 \\
j \neq i}}^{n}\left(1-p_{j}\right) \\
& +\sum_{i=1}^{n-1} p_{i} \sum_{j=i+1}^{n} p_{j} \prod_{\substack{k=1 \\
k \neq i, j}}^{n}\left(1-p_{k}\right)\left(R_{i}+R_{j}\right) \bar{A}\left(R_{i}, R_{j}\right) .
\end{aligned}
$$

We can also use the single user optimal rates characterized in (10) as upper bounds to the optimal rates due to the fact that capacity region of two-user channel is inside the rectangular capacity region determined by the single user capacities. Convergence to a globally optimal set of rates is then guaranteed by the following result whose proof is given in Appendix B.

Proposition 1: Let $R_{1}^{*}, R_{2}^{*}, . ., R_{n}^{*}$ be a locally optimal solution of the following problem:

$$
\max _{R_{1}, R_{2}, . ., R_{n}} \widetilde{T}_{M U D} \text { s.t. } 0 \leq R_{i} \leq R^{*}\left(d_{i}\right)=\frac{W\left(\frac{P_{0}}{N d_{i}^{\gamma}}\right)}{\ln (4)}, \quad \forall i .
$$

If condition $\mathbf{C} 1$ below holds, then $R_{1}^{*}, R_{2}^{*}, \ldots, R_{n}^{*}$ are also globally optimal.

We state the condition $\mathbf{C 1}$ on the user distances, (i.e., user SNRs) as follows.

$$
\text { C1: } \begin{aligned}
C\left(e^{-\alpha_{i} \sigma^{*}}-e^{-\alpha_{j} \sigma^{*}}\right)+\ln 2\left(R^{*}\left(d_{i}\right)+R^{*}\left(d_{j}\right)\right) \\
\quad \times\left[\frac{\alpha_{j} C-1}{\alpha_{j}} e^{-\alpha_{i} \sigma^{*}}-\frac{\alpha_{i} C-1}{\alpha_{i}} e^{-\alpha_{j} \sigma^{*}}\right] \geq 0, \quad \forall i, j,
\end{aligned}
$$

where $C=W\left(1 / \alpha_{i}\right) W\left(1 / \alpha_{j}\right)$ and $\sigma^{*}=2^{2\left(R^{*}\left(d_{i}\right)+R^{*}\left(d_{j}\right)\right)}-1$. We note that this technical condition is not very restrictive in practical scenarios. For instance, in the low power long range setups (as in LoRaWAN and NB-IoT), it is satisfied with a high probability.

Under condition $\mathbf{C 1}$, by using this proposition, we can obtain a globally optimal set of rates with iterative algorithms using the modified problem in (28), which is a relaxed version of the original problem. Via extensive numerical examples, we have observed that the solution of the modified problem in (28) closely matches with the solution of the original problem in (27). Therefore, we can argue that the algorithm that we use to solve the problem (27) converges to a point close to the globally optimal one (at least for the extensive set of examples considered) due to the fact that the modified problem is a convex relaxation of the original problem. Alternatively, we can argue that the modified problem in (28) can be solved to find the optimal rates, where we can use algorithms that guarantee convergence to the globally optimal points ${ }^{7}$. In the cases where condition $\mathbf{C 1}$ does not hold, we observe that the algorithm solving (28) still performs quite well even though we cannot guarantee a globally optimal result analytically. We provide an intuitive explanation of this favorable behavior in the last part of Appendix B.

\section{B. System Design With Fairness}

In highly asymmetric scenarios where some users are much closer to the destination than the others, individual throughputs of far away users become very low. With this motivation, we now consider an extension of the proposed scheme for the case with MUD by also taking into account fairness among users. The main difference with the case with no MUD is that the optimal rates also change with activity probabilities and rates of the other users. Therefore, they cannot be taken as constants, i.e., they are parameters in the optimization problem as well. Specifically, we impose a minimum individual throughput constraint and propose a method to compute the optimal rates and activity probabilities. Namely, we formulate

$$
\begin{aligned}
& \max _{\substack{p_{1}, p_{2}, \ldots, p_{n} \\
R_{1}, R_{2}, \ldots, R_{n}}} \sum_{i=1}^{n} p_{i} \prod_{\substack{j=1 \\
j \neq i}}^{n}\left(1-p_{j}\right) R_{i} \exp \left(-\alpha_{i}\left(2^{2 R_{i}}-1\right)\right) \\
& \quad+\sum_{i=1}^{n-1} p_{i} \sum_{j=i+1}^{n} p_{j} \prod_{\substack{k=1 \\
k \neq i, j}}^{n}\left(1-p_{k}\right)\left(R_{i}+R_{j}\right) A\left(R_{i}, R_{j}\right) \\
& \text { s.t. } R_{i}\left(p_{i} \prod_{\substack{j=1 \\
j \neq i}}^{n}\left(1-p_{j}\right) \exp \left(-\alpha_{i}\left(2^{2 R_{i}}-1\right)\right)\right. \\
& \left.+p_{i} \sum_{\substack{j=1 \\
j \neq i}}^{n} p_{j} \prod_{\substack{k=1 \\
k \neq i, j}}^{n}\left(1-p_{k}\right) A\left(R_{i}, R_{j}\right)\right) \geq K, \\
& 0 \leq p_{i} \leq 1, \quad R_{i} \geq 0 \text { for } i=1,2, \ldots, n .
\end{aligned}
$$

${ }^{7}$ If a gradient descent based algorithm is used to solve (27), it can be modified to a projected gradient descent algorithm in a straightforward manner to solve (28), where projection onto feasible set is one dimensional operation for each user's rate $R_{i}$. 
Here $K$ is the minimum throughput required for each user. The optimal rates $R_{i}^{*}$ and activity probabilities $p_{i}^{*}$ are then determined via the following steps:

1) Set the (feasible) initial values for $R_{i}$ 's and the objective function value $T_{\text {fair }}$ as zero.

2) Fix $R_{i}$ 's and find the optimal activity probabilities $p_{i}^{*}$ 's via an interior-point method, and update $p_{i}$ 's with the newly found values.

3) Fix $p_{i}$ 's and find the optimal rates $R_{i}^{*}$ 's via an interiorpoint method, and update $R_{i}$ 's with the newly found values. Calculate $T_{\text {fair }}^{*}$ with the current values of $p_{i}$ 's and $R_{i}$ 's using (24).

4) Examine whether $T_{\text {fair }}^{*}-T_{\text {fair }}$ is higher than some small tolerance $\epsilon$. If so, return to step 2 and update $T_{\text {fair }}$ with $T_{\text {fair }}^{*}$. If not, stop and set as the optimal rates and activity probabilities $R_{i}$ and $p_{i}$, respectively.

This method is guaranteed to converge, however, the solution may be locally optimal. This is because, the objective function increases at each iteration, and it is bounded from above. It can also be noted that, the optimal rates and activity probabilities can be found jointly in one step, however, the proposed step by step approach helps reduce the computational complexity significantly, hence it is more practical.

\section{Limiting Individual Outage Probabilities}

Similar to the case where MUD is not performed at the receiver side (discussed in Section III-C), we also consider limiting the individual outage probabilities for the present scheme.

We formulate the corresponding optimization problem as

$$
\begin{aligned}
& \max _{R_{1}, R_{2}, ., R_{n}} \sum_{i=1}^{n} p_{i} \prod_{\substack{j=1 \\
j \neq i}}^{n}\left(1-p_{j}\right) R_{i} \exp \left(-\alpha_{i}\left(2^{2 R_{i}}-1\right)\right) \\
&+\sum_{i=1}^{n-1} p_{i} \sum_{j=i+1}^{n} p_{j} \prod_{\substack{k=1 \\
k \neq i, j}}^{n}\left(1-p_{k}\right)\left(R_{i}+R_{j}\right) A\left(R_{i}, R_{j}\right) \\
& \text { s.t. } 1-\exp \left(-\alpha_{i}\left(2^{2 R_{i}}-1\right)\right) \leq \beta_{i}, 1-A\left(R_{i}, R_{j}\right) \leq \beta_{i}, \quad \forall j \\
& \quad 0 \leq p_{i} \leq 1, \quad R_{i} \geq 0 \text { for } i=1,2, \ldots, n,
\end{aligned}
$$

where $\beta_{i}$ is the maximum allowed individual outage probability for each user. Given feasible $\beta_{i}$ 's and the activity probabilities $p_{i}$ 's, the optimal rates $R_{i}^{*}$ 's can again be found via numerical algorithms such as gradient descent or interiorpoint methods. Hence, the proposed formulation provides a way to design systems with guaranteed individual outage probabilities. ${ }^{8}$ We observe that under this formulation, the optimal rates and the optimum throughput decrease in order to satisfy the additional constraints introduced.

\section{Practical CONSIDERATIONS}

Throughout the paper, we assume that the receiver knows the identities of active users in a slot. We note that this is a

\footnotetext{
${ }^{8}$ We note that the constraints in (29) can also be included in the formulation here in order to combine fairness considerations and limitation on the individual outage probabilities.
}

common assumption in related works as well [16], [17], [22], [31], and it can be accomplished by transmitting sufficiently coded identifying tags added to each packet [16], ${ }^{9}$ by using a separate control channel [22], and by a method based on random set theory [33].

CSI estimation at the receiver is also not very straightforward with two users in the same slot due to the need of separation of the packets and obtaining reliable channel estimation for both of them. A solution to this problem can be obtained by sending additional $\ell$ bits for receiver to estimate the channel state. That is, each user sends unique $\ell$ bits sequences such that any two pair of users have at least one common and one different symbol. It is possible to obtain sets of $2^{\ell-1}$ different sequences following this rule. With this way, for channel states $h_{i}$ and $h_{j}$, the receiver obtains $h_{i}+h_{j}$ and $h_{i}-h_{j}$, which is enough to determine them individually. Therefore, by sending additional $\ell$ bits, we can support $2^{\ell-1}$ users.

Optimum rate of a user is independent from the other user's rates, activity probabilities, locations and channel states when MUD is not considered, as shown in solutions in (10) and (23). This makes the solution attractive since it can be implemented in a decentralized manner. That is, any user can compute its own optimal rate with (10) by using its own distance to the receiver and an estimation of path-loss exponent without needing any information and/or statistics from the others. Therefore, there is no need for a feedback link from the receiver to the transmitter. On the other hand, when MUD is considered, the proposed optimization procedure is centralized. That is, we assume that the distances of all the users to the receiver are known, which increases the system complexity. Namely, the optimization process should be repeated for each update on the distances, and the receiver should use a feedback link to notify the users on the new optimal rates. A strategy to reduce the necessary feedback can be grouping the users based on their distances and feeding back their optimal rates (which are common for all the users in a group) only if there is a significant change in them.

To reduce the receiver complexity and eliminate the need for the feedback link fully, as an alternative, the system design can be performed by using only the statistical knowledge of user locations. We formulate the optimization procedure with the distance statistics rather than their exact values as follows

$$
\begin{aligned}
& \max _{R(d)} \int_{d_{\min }}^{d_{\max }} f_{d}\left(d_{i}\right) p(1-p)^{n-1} R\left(d_{i}\right) \exp \left(-\alpha_{i}\left(2^{2 R\left(d_{i}\right)}-1\right)\right) \mathrm{d} d_{i} \\
& \quad+\int_{d_{\min }}^{d_{\max }} f_{d}\left(d_{j}\right) \int_{d_{j}}^{d_{\max }} f_{d}\left(d_{i}\right) p^{2}(1-p)^{n-2}\left(R\left(d_{i}\right)+R\left(d_{j}\right)\right) \\
& \quad \times A\left(R\left(d_{i}\right), R\left(d_{j}\right)\right) \mathrm{d} d_{i} \mathrm{~d} d_{j}
\end{aligned}
$$

where we assume $p_{i}=p, \forall i=1,2, \ldots, n$. Here $f_{d}(\cdot)$ denotes p.d.f. of the user distances to the receiver where $R\left(d_{i}\right)$ and $R\left(d_{j}\right)$ are different realizations of the same function $R(d)$

\footnotetext{
${ }^{9}$ Even though most works assume the cost of identifying tags is negligible, this might still create an overhead for small data communication with large number of users. In order to reduce the overhead, a possible solution is adopted in [32], in which a user's first packet includes a random seed which makes possible to reconstruct locations of its other packets with a pre-defined pseudorandom generator.
} 


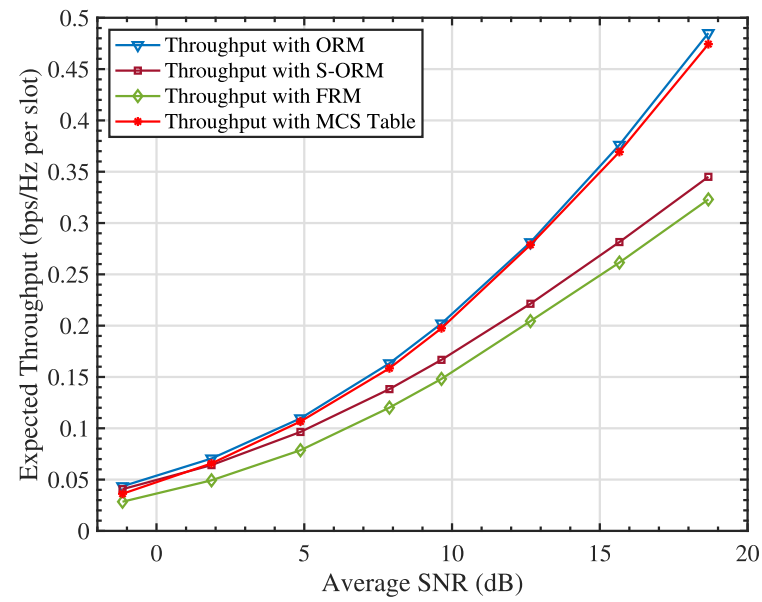

Fig. 3. Performance comparison for different rate selections (with $\gamma=3$, $n=40000$ and $p_{i}=1 / 40000$ for all the users that are distributed uniformly on a ring with $d_{\min }=200 \mathrm{~m}$ and $d_{\max }=1000 \mathrm{~m}$ ).

at distances $d_{i}$ and $d_{j}$, respectively ${ }^{10}$. Noting that various constraints regarding fairness or individual outage probabilities can be included in a straightforward manner, we define the solution of (30) as optimal rate selection curve $R^{*}(d)$. The users can choose their rates using their only own distances by this pre-determined function. This approach is practical and effective as the number of users in the system is assumed large.

We can solve (30) for a particular distribution using the proposed method in (27) with user distance realizations specified by this distribution for a large number of users. An exemplary optimal rate selection curve $R^{*}(d)$ is given in [1, Ch. 4.6.1] for the case where the user distances are distributed uniformly between distances $d_{\min }$ and $d_{\max }$.

\section{NUMERICAL EXAMPLES}

We first consider slotted ALOHA with SUD only and provide a comparison among the throughput performances of various rate selection schemes in Fig. 3. We set $\gamma=3$, $n=40000$ and $p_{i}=1 / 40000$ for all the users, and assume that they are distributed uniformly on a ring of inner radius $d_{\min }=200 \mathrm{~m}$ and outer radius $d_{\max }=1000 \mathrm{~m}$. We name the scheme obtained by (10) as the optimal rate method (ORM), the approach that uses the rates $R\left(d_{i}\right)=\frac{1}{2} \log _{2}\left(1+\frac{P_{i}}{N}\right)$ where $P_{i}$ is the average received power of user $i$ as the suboptimal rate method (S-ORM), and the one using the same rate for all the users (equal to the average channel capacity) as the fixed rate method (FRM). In addition, we consider the case in which the users pick their rates from a finite set, i.e., from the modulation and coding scheme (MCS) table of IEEE 802.11.ac [35] protocol $^{11}$. The results clearly show that the proposed solution (ORM) is highly superior compared to FRM and S-ORM in terms of expected throughput, especially, for high average SNR values. We also observe that using the optimal rates from the MCS table performs very close to the ORM scheme, which is a desired behavior in practice,

\footnotetext{
${ }^{10}$ It is not easy to simplify the expression in (30) at this point, however, one can reformulate a similar problem with stochastic geometry [34], and try to obtain a closed-form result.

${ }^{11}$ The optimal rate of each user is found via exhaustive search.
}
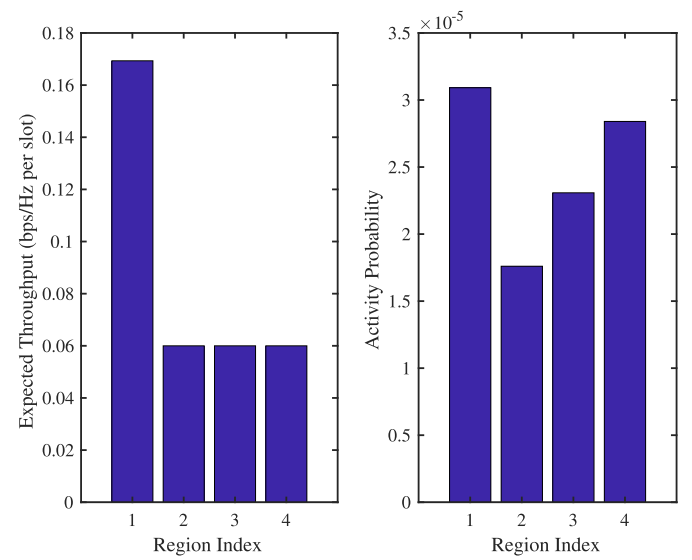

Fig. 4. A simulation of a fair system with equally populated four groups of users (with $\gamma=3, d_{1}=468 \mathrm{~m}, d_{2}=688 \mathrm{~m}, d_{3}=832 \mathrm{~m}, d_{4}=948 \mathrm{~m}$, $n=40000$, average SNR $=8.7 \mathrm{~dB}$ ).

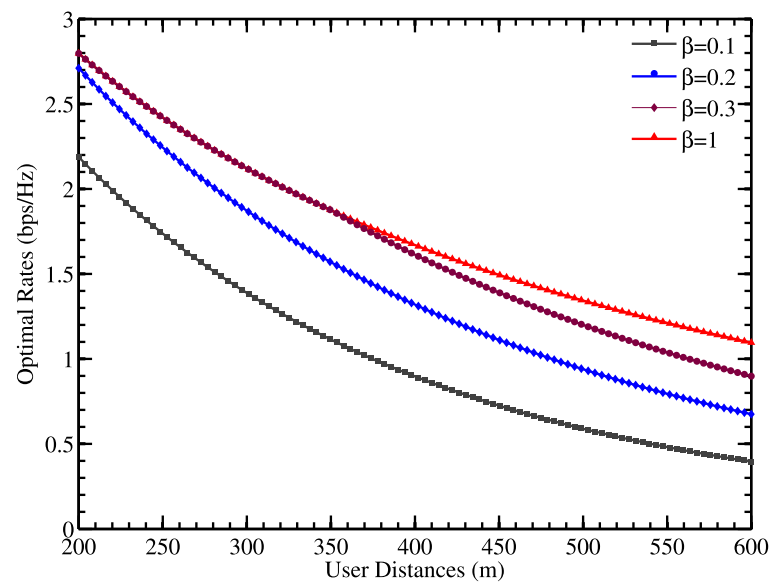

Fig. 5. Optimal rates with limited maximum outage probabilities (with $\gamma=$ $3, d_{\text {min }}=200 \mathrm{~m}, d_{\max }=600 \mathrm{~m}$, average $\mathrm{SNR}=8.7 \mathrm{~dB}$ ).

indicating that a quantized version of ORM can be used effectively.

We provide an example of system design with fairness for $k=4$ distinct group of users in Fig. 4 . We set the group load $\lambda_{j}$ as in (17), and the minimum group throughput as $K=0.06$. The plot on the left hand side shows the throughput of users in each region while the one on the right hand side shows the optimal activity probabilities characterized in (17). Clearly, far away users send their packets more frequently than the closer ones. We also note that Jain's fairness index [36], which can be used as a quantitative measure of the fairness among different users, is calculated as 0.771 for this example. If an application requires more fairness, one can increase the value of $K$, or even use the solutions in (18) for which the maximum achievable Jain's index of 1 will be attained.

In order to illustrate a design with limited outage probabilities, we use a setup with $n=100$ users and four different outage probability limits (taken the same for all the users as $\beta=0.1,0.2,0.3,1)$. The resulting optimal rates and outage probabilities corresponding to them are given in Figs. 5 and 6 , respectively. We observe that, for stricter outage limits (i.e., smaller $\beta$ ), one should decrease the transmission rates causing lower expected individual throughputs for the users. 


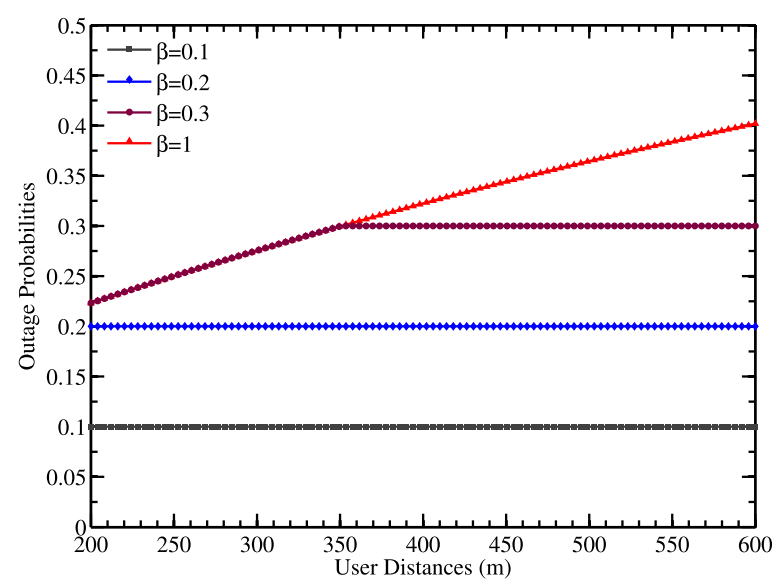

Fig. 6. Outage probabilities vs. user distances for different outage thresholds (with $\gamma=3, d_{\min }=200 \mathrm{~m}, d_{\max }=600 \mathrm{~m}$, average SNR $=8.7 \mathrm{~dB}$ ).

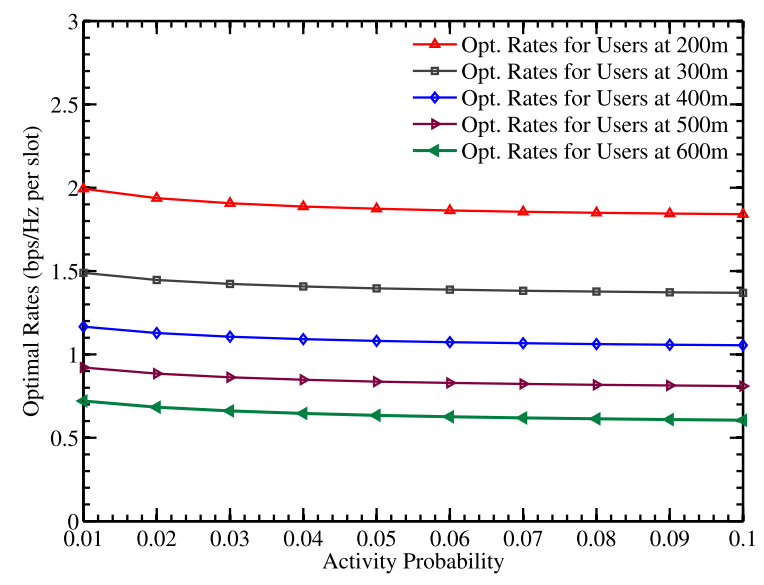

Fig. 7. Optimal rates for 5 groups of users with MUD.

If an application requires high expected throughputs, then the system can be designed with a loose outage probability policy (i.e., with a large $\beta$ ).

We now turn our attention to the case of slotted ALOHA with MUD. We consider 5 groups of users placed at distances of $200 \mathrm{~m}, 300 \mathrm{~m}, 400 \mathrm{~m}, 500 \mathrm{~m}$ and $600 \mathrm{~m}$. We assume that each group has 20 users, and we set the path-loss exponent $\gamma=3$, and the average SNRs of groups $1-5$ as $22.6 \mathrm{~dB}, 17.3 \mathrm{~dB}$, $13.6 \mathrm{~dB}, 10.7 \mathrm{~dB}, 8.4 \mathrm{~dB}$, respectively. Fig. 7 shows the resulting optimal rates in the symmetric scenario of equal activity probabilities. The optimal rates decrease with increasing activity probabilities as expected from the fact that the probability of collisions increase as user activity increases.

Comparisons among the throughput performance ${ }^{12}$ of our model and the one with the same rate assignment to all the users are presented in Fig. 8 for both SUD and MUD. We observe that the newly proposed method outperforms the results of the same rate to all users approach for both scenarios. We also note that, if the users are more spatially separated,

\footnotetext{
${ }^{12}$ We note that the expected throughput is evaluated with simulations where decoders are implemented based on the information-theoretic models.
}

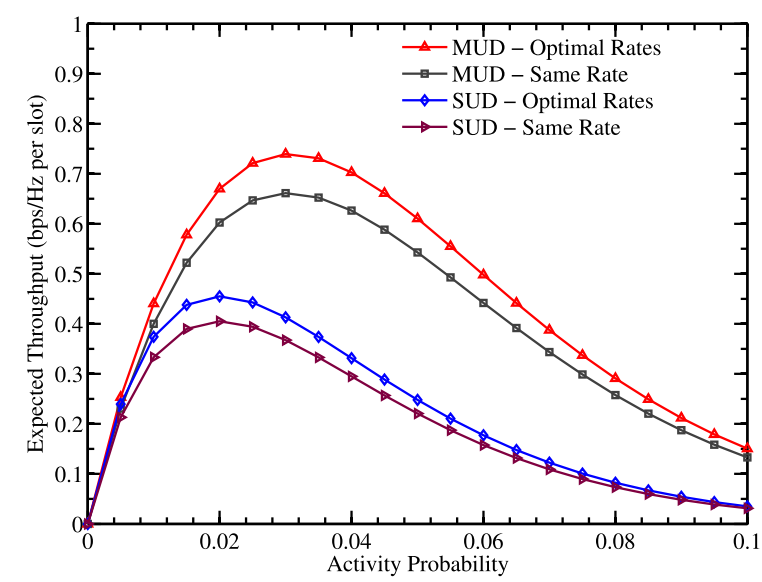

Fig. 8. Expected throughput for different setups with $n=50$. User distances are distributed uniformly between $d_{\min }=200 \mathrm{~m}$ and $d_{\max }=600 \mathrm{~m}$.

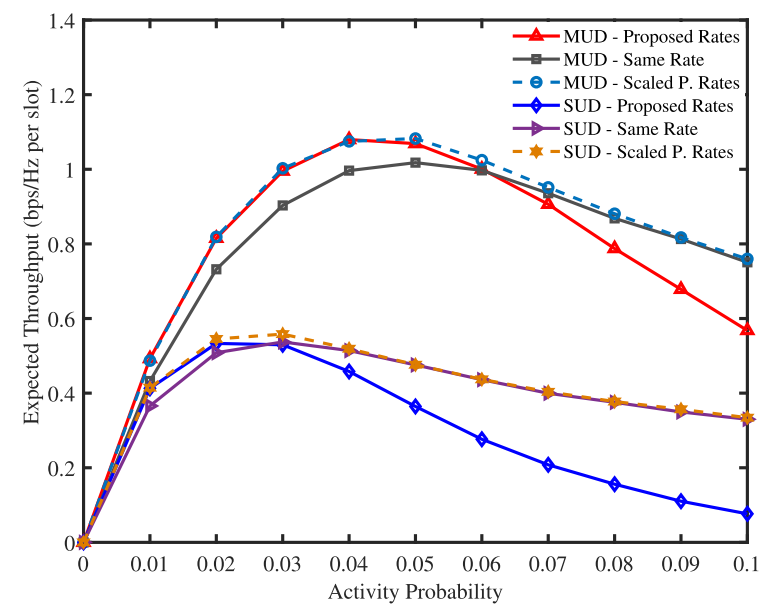

Fig. 9. Performance evaluation with a decoder with capture.

the gains will increase further, since proposed methods exploit the asymmetry among user distances.

In addition, for the same setup, we evaluate the performance of the proposed method for decoding with capture. In this scenario, the decoder tries joint decoding first and if this is impossible, it then tries decoding of stronger signals by considering the others as noise. Therefore, if there are more than two active users, they are not necessarily assumed as lost. The results are shown in Fig. 9. We observe that the newly optimized rates result in a better throughput than the same rate to all users approach in low activity probabilities. However, in higher activity probabilities their performance deteriorates. This is because, for the latter, the need for capture increases, and signal to interference plus noise ratio (SINR) becomes as important as SNR, implying that the rates should be reduced for improved performance since they have been optimized only considering the expected SNR values. As an heuristic approach, we can scale down the rates for higher activity probabilities, where the scaling factor is found with a grid search for different activity probabilities. More specifically, we first find the optimal rates $R_{i}^{*}$ with the proposed methods solving (27), and then obtain $\rho^{*}=\arg \max _{\rho} T_{\text {tot }}\left(\hat{R}_{1}, \ldots, \hat{R}_{n}\right)$ 


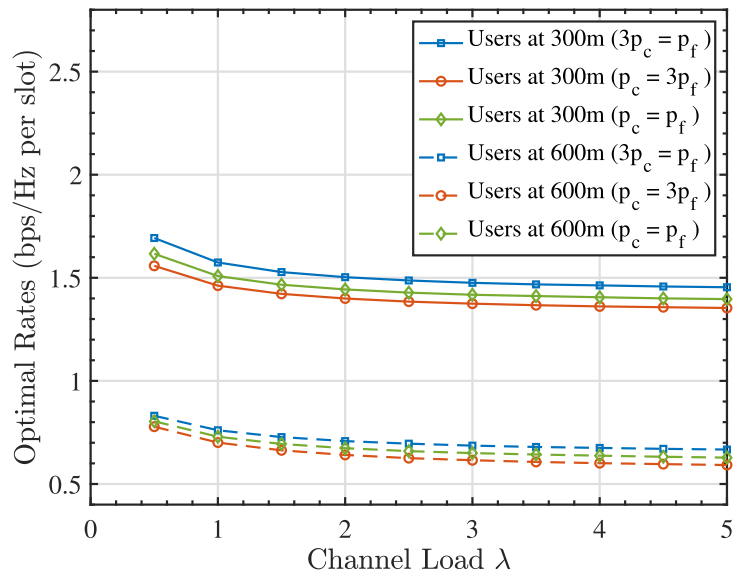

Fig. 10. Optimal rates with various activity probability scenarios.

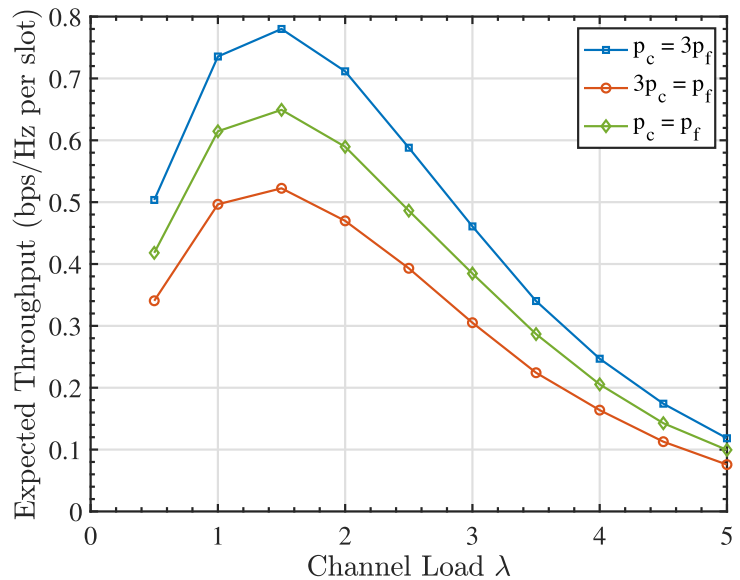

Fig. 11. Expected throughput for different setups with $n=50$. The user distances are distributed uniformly between $d_{\min }=200 \mathrm{~m}$ and $d_{\max }=$ $600 m$.

maximizing the total throughput, where $\hat{R}_{i}=\rho R_{i}^{*}$, via a grid search on $\rho \in[0,1]$. Here, $T_{\text {tot }}$ denotes the total throughput obtained through simulations with the capture effect. With scaling, the performance becomes slightly better than the same rate to all users approach. To reiterate, our results show that, despite being sub-optimal, it is beneficial to use the newly optimized rates especially in low activity probabilities for the case of decoding with capture, for which determination of the optimal rates is highly challenging when the number of the users is relatively large.

It is also important to investigate the cases where the activity probabilities of the users are not identical. To exemplify this case, we consider two groups of users placed at distances of $300 \mathrm{~m}$ and $600 \mathrm{~m}$, where each group has $n_{c}=n_{f}=50$ users. The closer group's activity probabilities are drawn from a uniform distribution between $\left[0.9 p_{c}, 1.1 p_{c}\right]$, and the other group's activity probabilities are drawn from a uniform distribution between $\left[0.9 p_{f}, 1.1 p_{f}\right]$, where the channel load is $\lambda=n_{c} p_{c}+n_{f} p_{f}$. Average SNR's of the groups are $17.3 \mathrm{~dB}$ and $8.4 \mathrm{~dB}$. Fig. 10 presents the optimal rates obtained by solving (27) for various activity probability scenarios. As shown, optimal rates increase when the activity probabilities of the closer users get higher. In addition, as shown in Fig. 11,
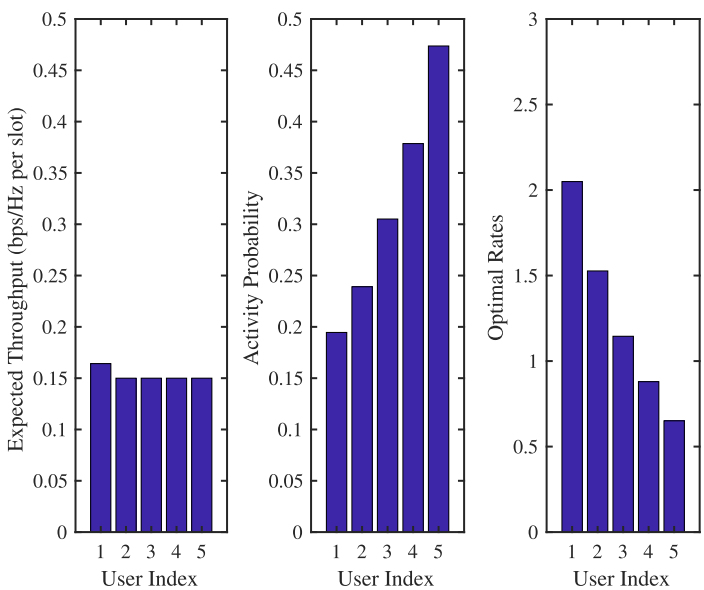

Fig. 12. A simulation of a fair system with MUD for $K=0.15$ (with $\gamma=3, d_{1}=200 \mathrm{~m}, d_{2}=300 \mathrm{~m}, d_{3}=400 \mathrm{~m}, d_{4}=500 \mathrm{~m}, d_{5}=600 \mathrm{~m}$, average $\mathrm{SNR}=8.7 \mathrm{~dB}$ ).
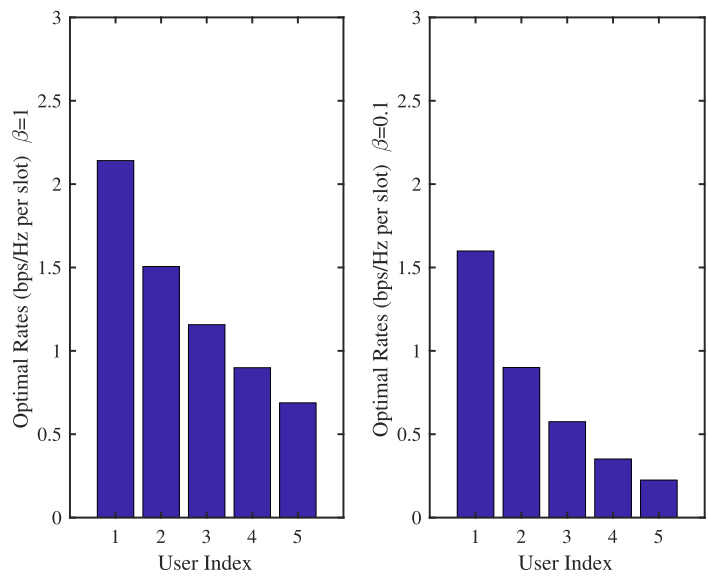

Fig. 13. System design with limited outage probabilities (with $\gamma=3$, $d_{1}=200 \mathrm{~m}, d_{2}=300 \mathrm{~m}, d_{3}=400 \mathrm{~m}, d_{4}=500 \mathrm{~m}, d_{5}=600 \mathrm{~m}, p_{i}=$ $0.2, \forall i=1,2, \ldots, n$, average $\mathrm{SNR}=8.7 \mathrm{~dB}$ ).

the expected throughput increases when the group with the higher mean SNR becomes more active.

As a final example, we evaluate the optimal rates with fairness and limited individual outage probabilities. For exposition purposes, we use a simple setup with 5 users with distances to the receiver $200 \mathrm{~m}, 300 \mathrm{~m}, 400 \mathrm{~m}, 500 \mathrm{~m}$ and $600 \mathrm{~m}$ and $\gamma=3$. For the fairness example, we set the minimum individual throughput threshold to $K=0.15$. In the MUD case, the optimization is performed not only over the activity probabilities but also over the rates as characterized in (29). As shown in Fig. 12, our solution assigns higher activity probabilities to the far away users to maintain the minimum required individual throughput. After satisfying the minimum throughput constraints for each user, it is optimal to favor the closest one since it has the largest SNR. In Fig. 13, on the left hand side, we take $\beta=1$ which means that we do not limit the outage probabilities, while on the right hand side we take $\beta=0.1$ which means that no outage probability can exceed 0.1 . Clearly, limiting the outage probabilities decreases the optimal rates similar to the case of SUD. We also note that the 
optimal rate of a user for the MUD case depends on the other users' outage constraints since we perform joint optimization as opposed to the single-user scenario where the design can be decomposed into separate optimization problems.

\section{CONCLUSION}

In this paper, we have proposed methods for obtaining optimal rates and activity probabilities for random access networks over wireless block fading channels. We have obtained closedform solutions for the classical slotted ALOHA framework, while we have resorted to numerical optimization approaches to find the optimal operating parameters for the system with MUD. In addition, we have modified our methods to adopt additional constraints of fairness and/or limited individual outage probabilities. Our results indicate that with the proposed optimization framework, which allows for unequal transmission rates while providing fairness, the expected throughputs can be improved significantly compared to the existing solutions especially in the long range applications. A future direction of this work can be investigation of optimal rate allocations with the recently developed schemes like CRDSA [31] and IRSA [37]. It would also be interesting to optimize the MCS tables for the set of rates that can be used in practice.

\section{APPENDIX A}

Global Optimality of The Solution In (17)

In this section, we verify the global optimality of the solution in (17). We denote the system throughput as $T=$ $\left(e^{-\sum_{i=1}^{k} \lambda_{i}}\right) \sum_{j=1}^{k} \lambda_{j} r_{j}$ and define $T^{\prime}=-\log (T)$, where $\log (\cdot)$ denotes natural logarithm function. Then, we have

$$
T^{\prime}=\sum_{i=1}^{k} \lambda_{i}-\log \left(\sum_{j=1}^{k} \lambda_{j} r_{j}\right) .
$$

We can rewrite (31) using matrix notation as $T^{\prime}=\mathbf{1}^{T} \boldsymbol{\lambda}-$ $\log \left(\mathbf{r}^{T} \boldsymbol{\lambda}\right)$ where 1 is the column vector of all 1's. $\mathbf{r}$ and $\boldsymbol{\lambda}$ are column vectors of $r_{i}$ 's and $\lambda_{i}$ 's, respectively. Here $(\cdot)^{T}$ denotes transpose operation. Denoting gradient with $\nabla$ and Hessian with $\nabla^{2}$, we can write

$$
\nabla T^{\prime}(\boldsymbol{\lambda})=\mathbf{1}^{T}-\frac{\mathbf{r}^{T}}{\mathbf{r}^{T} \boldsymbol{\lambda}}, \quad \nabla^{2} T^{\prime}(\boldsymbol{\lambda})=\frac{\mathbf{r r}^{T}}{\left(\mathbf{r}^{T} \boldsymbol{\lambda}\right)^{2}}
$$

where Hessian matrix $\nabla^{2} T^{\prime}(\boldsymbol{\lambda})$ is positive semidefinite. Therefore, $\log (T)$ is concave, and the system throughput $T$ is log-concave.

Since all of our variables are nonnegative, we can rewrite the problem in (12) as a convex optimization problem as follows

$$
\begin{aligned}
& \max _{\lambda_{1}, \lambda_{2}, \ldots, \lambda_{k} \geq 0} \log \left(\left(e^{-\sum_{i=1}^{k} \lambda_{i}}\right) \sum_{j=1}^{k} \lambda_{j} r_{j}\right) \\
& \text { s.t. } \log \left(\left(e^{-\sum_{i=1}^{k} \lambda_{i}}\right) \lambda_{j} r_{j}\right) \geq \log (K), \quad j=1,2, . ., k,
\end{aligned}
$$

where we have a concave objective function and convex constraint sets for $j=1,2, \ldots, k$. The optimal solution of the convex problem in (33) is actually the same result obtained in (17), and therefore, the solution in (17) is globally optimal.

\section{APPENDIX B \\ PROOF OF THE PROPOSITION 1}

In this appendix, we prove concavity of the modified problem in (28) by characterizing the domain where the objective function is concave and verifying the fact that constraint set is always in this domain under condition $\mathbf{C 1}$. We write the system throughput $\widetilde{T}_{M U D}$ as a summation of single user throughputs $T^{(i)}$ 's and two-user collision throughputs $\widetilde{T}_{i j}$ 's. Namely,

$$
\widetilde{T}_{M U D}=\sum_{i=1}^{n} T^{(i)}+\sum_{i=1}^{n-1} \sum_{j=i+1}^{n} \widetilde{T}_{i j}
$$

where $T^{(i)}=p_{i} \prod_{\substack{j=1 \\ j \neq i}}^{n}\left(1-p_{j}\right) R_{i} e^{-\alpha_{i}\left(2^{2 R_{i}}-1\right)}$ and $\widetilde{T}_{i j}=$ $p_{i} p_{j} \prod_{\substack{k=1 \\ k \neq i, j}}^{n}\left(1-p_{k}\right)\left(R_{i}^{j \neq i}+R_{j}\right) \bar{A}\left(R_{i}, R_{j}\right)$.

In Section III-A, we have proved that the solution obtained for single user throughput $T^{(i)}$ is globally optimal. The first derivative of $T^{(i)}$ with respect to $R_{i}$ is analyzed in (11), and at the optimal rates, from (9) we have

$$
1-2^{2 R^{*}\left(d_{i}\right)} \alpha_{i} 2 R^{*}\left(d_{i}\right) \ln 2=0 .
$$

The second derivative is given by

$$
\begin{gathered}
\frac{\partial^{2}\left(-T^{(i)}\right)}{\partial R_{i}^{2}}=e^{-\left(2^{2 R_{i}}-1\right) \alpha_{i}} p_{i} \prod_{\substack{j=1 \\
j \neq i}}^{n}\left(1-p_{j}\right) 2^{2 R_{i}} 4 \alpha_{i} \ln 2 \\
\times\left(1-2^{2 R_{i}} \alpha_{i} R_{i} \ln 2+R_{i} \ln 2\right) .
\end{gathered}
$$

For $\alpha_{i} \neq \alpha_{j}$, we have the first derivative of $-\widetilde{T}_{i j}$ as

$$
\begin{gathered}
\frac{\partial\left(-\widetilde{T}_{i j}\right)}{\partial R_{i}}=\frac{\phi_{i j}}{\alpha_{i}-\alpha_{j}}\left[\alpha_{j} e^{-\alpha_{i} \sigma}-\alpha_{i} e^{-\alpha_{j} \sigma}-2^{2\left(R_{i}+R_{j}\right)} \alpha_{i} \alpha_{j}\right. \\
\left.\times 2\left(R_{i}+R_{j}\right) \ln 2\left(e^{-\alpha_{i} \sigma}-e^{-\alpha_{j} \sigma}\right)\right]
\end{gathered}
$$

with $\sigma=2^{2\left(R_{i}+R_{j}\right)}-1$ and $\phi_{i j}=p_{i} p_{j} \prod_{\substack{k=1 \\ k \neq i, j}}^{n}\left(1-p_{k}\right)$.

For the second derivative, we obtain

$$
\begin{aligned}
\frac{\partial^{2}\left(-\widetilde{T}_{i j}\right)}{\partial R_{i}^{2}} & \\
= & \frac{\partial^{2}\left(-\widetilde{T}_{i j}\right)}{\partial R_{j}^{2}}=\frac{\partial^{2}\left(-\widetilde{T}_{i j}\right)}{\partial R_{i} \partial R_{j}} \\
= & \frac{\phi_{i j}}{\alpha_{i}-\alpha_{j}}\left(\left(-2\left(\sigma_{1} \alpha_{i} \alpha_{j} \sigma_{3} \ln (2)-\sigma_{1} \alpha_{i} \alpha_{j} \sigma_{2} \ln (2)\right)\right)\right. \\
& -\left(2^{2\left(R_{i}+R_{j}\right)+2} \alpha_{i} \alpha_{j} \ln (2)^{2}\left(R_{i}+R_{j}\right)\right. \\
& \left.\left.\times\left(\sigma_{3}-\sigma_{2}-\sigma_{4} \alpha_{i} \sigma_{3}+\sigma_{4} \alpha_{j} \sigma_{2}\right)\right)\right),
\end{aligned}
$$

where $\sigma_{1}=2^{2\left(R_{i}+R_{j}\right)+1}, \sigma_{2}=e^{-\alpha_{j}\left(\sigma_{4}-1\right)}, \sigma_{3}=e^{-\alpha_{i}\left(\sigma_{4}-1\right)}$ and $\sigma_{4}=2^{2\left(R_{i}+R_{j}\right)}$.

Lemma 1: The objective function $\widetilde{T}_{M U D}$ is concave in the region defined by

$$
\begin{aligned}
& 0 \leq R_{i}+R_{j} \leq M_{i j}, \quad \forall i, j=1,2, \ldots, n, \\
& 0 \leq R_{i} \leq M_{i}, \quad i=1,2, \ldots, n,
\end{aligned}
$$

where $M_{i j}$ and $M_{i}$ are positive constants for all $i, j=$ $1,2, \ldots, n$. 
Proof: We can write the $n \times n$ Hessian matrix of $-\widetilde{T}_{M U D}$ as

$$
\mathbf{H}=\left[\begin{array}{cccc}
\frac{\partial^{2}\left(-\widetilde{T}_{M U D}\right)}{\partial R_{1}^{2}} & \frac{\partial^{2}\left(-\widetilde{T}_{12}\right)}{\partial R_{1} \partial R_{2}} & \cdots & \frac{\partial^{2}\left(-\widetilde{T}_{1 n}\right)}{\partial R_{1} \partial R_{n}} \\
\frac{\partial^{2}\left(-\widetilde{T}_{12}\right)}{\partial R_{1} \partial R_{2}} & \frac{\partial^{2}\left(-\widetilde{T}_{M U D}\right)}{\partial R_{2}^{2}} & & \frac{\partial^{2}\left(-\widetilde{T}_{1 n}\right)}{\partial R_{1} \partial R_{n}} \\
\vdots & & \ddots & \vdots \\
\frac{\partial^{2}\left(-\widetilde{T}_{1 n}\right)}{\partial R_{1} \partial R_{n}} & \cdots & & \frac{\partial^{2}\left(-\widetilde{T}_{M U D}\right)}{\partial R_{n}^{2}}
\end{array}\right]
$$

where $\frac{\partial^{2}\left(-\widetilde{T}_{M U D}\right)}{\partial R_{i}^{2}}=\frac{\partial^{2}\left(-T^{(i)}\right)}{\partial R_{i}^{2}}+\sum_{\substack{j=1 \\ j \neq i}}^{n} \frac{\partial^{2}\left(-\widetilde{T}_{i j}\right)}{\partial R_{i}^{2}}$ for $i=$ $1,2, \ldots, n$

Consider an arbitrary column vector $\mathbf{x}=\left(x_{1}, x_{2}, \ldots, x_{n}\right)^{T}$, and compute

$$
\begin{aligned}
\mathbf{x}^{T} \mathbf{H x} & x_{1}^{2}\left(\frac{\partial^{2}\left(-T^{(1)}\right)}{\partial R_{1}^{2}}+\frac{\partial^{2}\left(-\widetilde{T}_{12}\right)}{\partial R_{1}^{2}}+\cdots+\frac{\partial^{2}\left(-\widetilde{T}_{1 n}\right)}{\partial R_{1}^{2}}\right) \\
& +x_{1} x_{2} \frac{\partial^{2}\left(-\widetilde{T}_{12}\right)}{\partial R_{1} \partial R_{2}}+\cdots+x_{1} x_{n} \frac{\partial^{2}\left(-\widetilde{T}_{1 n}\right)}{\partial R_{1} \partial R_{n}} \\
& +x_{2}^{2}\left(\frac{\partial^{2}\left(-T^{(2)}\right)}{\partial R_{2}^{2}}+\frac{\partial^{2}\left(-\widetilde{T}_{21}\right)}{\partial R_{2}^{2}}+\cdots+\frac{\partial^{2}\left(-\widetilde{T}_{2 n}\right)}{\partial R_{2}^{2}}\right) \\
& +x_{2} x_{1} \frac{\partial^{2}\left(-\widetilde{T}_{21}\right)}{\partial R_{2} \partial R_{1}}+\cdots+x_{2} x_{n} \frac{\partial^{2}\left(-\widetilde{T}_{2 n}\right)}{\partial R_{2} \partial R_{n}} \\
& \quad \vdots \\
& +x_{n}^{2}\left(\frac{\partial^{2}\left(-T^{(n)}\right)}{\partial R_{n}^{2}}+\frac{\partial^{2}\left(-\widetilde{T}_{n 1}\right)}{\partial R_{n}^{2}}+\cdots+\frac{\partial^{2}\left(-\widetilde{T}_{n n-1}\right)}{\partial R_{n}^{2}}\right) \\
& +x_{n} x_{1} \frac{\partial^{2}\left(-\widetilde{T}_{n 1}\right)}{\partial R_{n} \partial R_{1}}+\cdots+x_{n} x_{n-1} \frac{\partial^{2}\left(-\widetilde{T}_{n n-1}\right)}{\partial R_{n} \partial R_{n-1}} .
\end{aligned}
$$

This can be simplified by using (36) and (38) as

$$
\begin{aligned}
& \mathbf{x}^{T} \mathbf{H x} \\
& =\frac{\partial^{2}\left(-\widetilde{T}_{12}\right)}{\partial R_{1} \partial R_{2}}\left(x_{1}+x_{2}\right)^{2}+\frac{\partial^{2}\left(-\widetilde{T}_{13}\right)}{\partial R_{1} \partial R_{3}}\left(x_{1}+x_{3}\right)^{2} \\
& \quad+\frac{\partial^{2}\left(-\widetilde{T}_{23}\right)}{\partial R_{2} \partial R_{3}}\left(x_{2}+x_{3}\right)^{2}+\cdots+\frac{\partial^{2}\left(-\widetilde{T}_{n n-1}\right)}{\partial R_{n-1} \partial R_{n}}\left(x_{n}+x_{n-1}\right)^{2} \\
& \quad+\frac{\partial^{2}\left(-T^{(1)}\right)}{\partial R_{1}^{2}} x_{1}^{2}+\frac{\partial^{2}\left(-T^{(2)}\right)}{\partial R_{2}^{2}} x_{2}^{2}+\cdots+\frac{\partial^{2}\left(-T^{(n)}\right)}{\partial R_{n}^{2}} x_{n}^{2}
\end{aligned}
$$

Clearly, if $\frac{\partial^{2}\left(-T^{(i)}\right)}{\partial R_{i}^{2}} \geq 0$ and $\frac{\partial^{2}\left(-\widetilde{T}_{i j}\right)}{\partial R_{i}^{2}} \geq 0$ for $i=1,2, \ldots, n$, then $\mathbf{x}^{T} \mathbf{H} \mathbf{x} \geq 0$, therefore the matrix $\mathbf{H}$ is positive semidefinite, and $-\widetilde{T}_{M U D}$ is convex and $\widetilde{T}_{M U D}$ is concave.

From (36), we observe that $T^{(i)}$ is concave in the domain $\left[0, M_{i}\right]$ since $\frac{\partial^{2}\left(-T^{(i)}\right)}{\partial R_{i}^{2}} \geq 0$ for $0 \leq R_{i} \leq M_{i}$ and $\frac{\partial^{2}\left(-T_{i}\right)}{\partial R_{i}^{2}}<0$ for $R_{i}>M_{i}$, where $M_{i}$ is a constant. From (38) and assuming $\alpha_{j}>\alpha_{i}$, for concavity, we also need ${ }^{13}$

$$
\begin{aligned}
& \left(e^{-\alpha_{i} \sigma}-e^{-\alpha_{j} \sigma}\right)+\ln 2\left(R_{i}+R_{j}\right)\left[\left(e^{-\alpha_{i} \sigma}-e^{-\alpha_{j} \sigma}\right)\right. \\
& \left.-\alpha_{i} e^{-\alpha_{i} \sigma} 2^{2\left(R_{i}+R_{j}\right)}+\alpha_{j} e^{-\alpha_{j} \sigma} 2^{2\left(R_{i}+R_{j}\right)}\right] \geq 0 .
\end{aligned}
$$

\footnotetext{
${ }^{13}$ For simplicity, we omit $\alpha_{i}=\alpha_{j}$ case where this case is almost impossible in practical scenarios since there are at least some small difference between two distances.
}

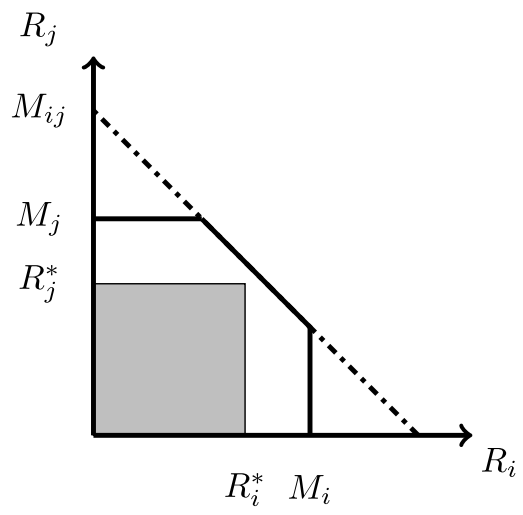

Fig. 14. Shaded region is the new constraint to guarantee a convex set of constraints in the optimization problem.

Note that (43) holds for $0 \leq R_{i}+R_{j} \leq M_{i j}$ and does not hold for $R_{i}+R_{j}>M_{i j}$ where $M_{i j}$ is a constant.

Lemma 2: Single user optimal rates, i.e., $R^{*}\left(d_{i}\right)=$ $W\left(\frac{P_{0}}{N d_{i}^{\gamma}}\right)$

$\frac{\ln (4)}{N d_{i}}, i=1,2, \ldots, n$ are inside of the concavity domain specified in (39) under condition $\mathbf{C 1}$.

Proof: By using (35) and the inequality (1 $\left.2^{2 R_{i}} \alpha_{i} R_{i} \ln 2+R_{i} \ln 2\right) \geq 0$ from (36) for concavity, we obtain

$$
1-2^{2 R^{*}\left(d_{i}\right)} \alpha_{i} R^{*}\left(d_{i}\right) \ln 2+R^{*}\left(d_{i}\right) \ln 2=1 / 2+R^{*}\left(d_{i}\right) \ln 2>0 .
$$

Hence, our optimal solution in (10) is inside the concavity domain $\left[0, M_{i}\right]$

We now investigate whether single user optimal rates are in this domain $\left[0, M_{i j}\right]$, i.e., we check if $R^{*}\left(d_{i}\right)+R^{*}\left(d_{j}\right) \leq M_{i j}$ holds. From (10), we have

$$
2^{2\left(R^{*}\left(d_{i}\right)+R^{*}\left(d_{j}\right)\right)}=\frac{1}{\alpha_{i} \alpha_{j} W\left(1 / \alpha_{i}\right) W\left(1 / \alpha_{j}\right)} .
$$

By defining $C=W\left(1 / \alpha_{i}\right) W\left(1 / \alpha_{j}\right)$ and from (43), for concavity, we need

$$
\begin{aligned}
C\left(e^{-\alpha_{i} \sigma}-\right. & \left.e^{-\alpha_{j} \sigma}\right)+\ln 2\left(R^{*}\left(d_{i}\right)+R^{*}\left(d_{j}\right)\right) \\
& \times\left[\frac{\alpha_{j} C-1}{\alpha_{j}} e^{-\alpha_{i} \sigma}-\frac{\alpha_{i} C-1}{\alpha_{i}} e^{-\alpha_{j} \sigma}\right] \geq 0,
\end{aligned}
$$

where it holds under condition $\mathbf{C 1}$, and the single user optimal rates $R^{*}\left(d_{i}\right)$ and $R^{*}\left(d_{j}\right)$ are inside this concavity domain.

Since the optimal rates are inside both domains $\left[0, M_{i}\right]$ and $\left[0, M_{i j}\right]$, our problem becomes convex in the domain $\left(R_{i}, R_{j}\right) \in\left[0, R^{*}\left(d_{i}\right)\right] \times\left[0, R^{*}\left(d_{j}\right)\right]$. Fig. 14 shows an illustration of these regions.

We know that the optimal rates with MUD are upper bounded by the single user rates $R^{*}\left(d_{i}\right)$ and $R^{*}\left(d_{j}\right)$ due to the fact that capacity region of two-user channel is inside of the intersection of the single user regions. Therefore, by using the constraints $R_{i} \leq R^{*}\left(d_{i}\right)=\frac{W\left(\frac{P_{0}}{N d_{i}^{\gamma}}\right)}{\ln (4)}$ for $i=1,2, \ldots, n$, we can guarantee that a suitable iterative optimization algorithm converges to the globally optimum solution for the modified version of the original problem. 
We note that we do not use condition $\mathbf{C 1}$ to show that $R^{*}\left(d_{i}\right) \leq M_{i}, \forall i$; in fact, this is true for every setup. Therefore, we can argue that in the scenarios where $\mathbf{C 1}$ does not hold, constraint set is not far away from concavity region even though we cannot guarantee it is inside the region. Therefore, the feasible iteration values are inside the concavity region with a high probability, which is a favorable behavior in terms of global optimality of the solutions.

\section{REFERENCES}

[1] N. Karakoc, "Random access over wireless links: Optimal rate and activity probability selection,” M.S. Thesis, Dept. Elect. Electron. Eng., Bilkent Univ., Ankara, Turkey, 2017.

[2] N. Karakoc and T. M. Duman, "Random access over wireless links: Optimal rate and activity probability selection," in Proc. GLOBECOM IEEE Global Commun. Conf., Dec. 2017, pp. 1-6.

[3] E. Paolini, C. Stefanovic, G. Liva, and P. Popovski, "Coded random access: Applying codes on graphs to design random access protocols," IEEE Commun. Mag., vol. 53, no. 6, pp. 144-150, Jun. 2015.

[4] F. Adelantado, X. Vilajosana, P. Tuset-Peiro, B. Martinez, J. Melia-Segui, and T. Watteyne, "Understanding the limits of LoRaWAN,” IEEE Commun. Mag., vol. 55, no. 9, pp. 34-40, Sep. 2017.

[5] M. Chen, Y. Miao, Y. Hao, and K. Hwang, "Narrow band Internet of Things," IEEE Access, vol. 5, pp. 20557-20577, 2017.

[6] LoRaWAN-What is it, A Technical Overview of LoRa and LoRaWAN, LoRa Alliance, San Ramon, CA, USA, 2015.

[7] A. Laya, C. Kalalas, F. Vazquez-Gallego, L. Alonso, and J. Alonso-Zarate, "Goodbye, ALOHA!," IEEE Access, vol. 4, pp. 2029-2044, 2016.

[8] Interaction Channel For Satellite Distribution Systems, document ETSI EN 301790, 2009.

[9] A. Augustin, J. Yi, T. Clausen, and W. M. Townsley, "A study of LoRa: Long range \& low power networks for the Internet of Things," Sensors, vol. 16 , no. 9 , p. 1466,2016

[10] N. Abramson, "The ALOHA system: Another alternative for computer communications," in Proc. Fall Joint Comput. Conf., Houston, TX, USA, Nov. 1970, pp. 281-285.

[11] L. G. Roberts, "ALOHA packet system with and without slots and capture," SIGCOMM Comput. Commun. Rev., vol. 5, no. 2, pp. 28-42, Apr. 1975.

[12] N. Abramson, "The throughput of packet broadcasting channels," IEEE Trans. Commun., vol. COM-25, no. 1, pp. 117-128, Jan. 1977.

[13] J. Metzner, "On improving utilization in ALOHA networks," IEEE Trans. Commun., vol. COM-24, no. 4, pp. 447-448, Apr. 1976.

[14] S. Verdú, Multiuser Detection, 1st ed. New York, NY, USA: Cambridge Univ. Press, 1998

[15] M. Berioli, G. Cocco, G. Liva, and A. Munari, "Modern random access protocols," FNT Netw., vol. 10, no. 4, pp. 317-446, 2016

[16] M. Médard, J. Huang, A. Goldsmith, S. Meyn, and T. Coleman, "Capacity of time-slotted ALOHA packetized multiple-access systems over the AWGN channel," IEEE Trans. Wireless Commun., vol. 3, no. 2, pp. 486-499, Mar. 2004.

[17] C. Wei, P. Fan, and K. Letaief, "On channel coding selection in time-slotted ALOHA packetized multiple-access systems over Rayleigh fading channels," IEEE Trans. Wireless Commun., vol. 7, no. 5, pp. 1699-1707, May 2008

[18] X. Qin and R. Berry, "Distributed approaches for exploiting multiuser diversity in wireless networks," IEEE Trans. Inf. Theory, vol. 52, no. 2, pp. 392-413, Feb. 2006.

[19] Y. Hu and A. Ribeiro, "Adaptive distributed algorithms for optimal random access channels," IEEE Trans. Wireless Commun., vol. 10, no. 8 , pp. 2703-2715, Aug. 2011.

[20] J. Goseling, M. Gastpar, and J. H. Weber, "Random access with physical-layer network coding," IEEE Trans. Inf. Theory, vol. 61, no. 7, pp. 3670-3681, Jul. 2015.

[21] J. Choi, "NOMA-based random access with multichannel ALOHA," IEEE J. Sel. Areas Commun., vol. 35, no. 12, pp. 2736-2743, Dec. 2017.

[22] P. Minero, M. Franceschetti, and D. N. C. Tse, "Random Access: An information-theoretic perspective," IEEE Trans. Inf. Theory, vol. 58, no. 2, pp. 909-930, Feb. 2012.

[23] H. S. Dhillon, H. Huang, H. Viswanathan, and R. A. Valenzuela, "Fundamentals of throughput maximization with random arrivals for M2M communications," IEEE Trans. Commun., vol. 62, no. 11, pp. 4094-4109, Nov. 2014.
[24] Y. Polyanskiy, "A perspective on massive random-access," in Proc. IEEE Int. Symp. Inf. Theory (ISIT), Jun. 2017, pp. 2523-2527.

[25] Y. Li and L. Dai, "Maximum sum rate of slotted ALOHA with successive interference cancellation," IEEE Trans. Commun., vol. 66, no. 11 , pp. 5385-5400, Nov. 2018.

[26] T. M. Cover and J. A. Thomas, Elements of Information Theory. Hoboken, NJ, USA: Wiley, 2006.

[27] T. Cui, T. Ho, and J. Kliewer, "Achievable rate and optimal physical layer rate allocation in interference-free wireless networks," in Proc. IEEE Int. Symp. Inf. Theory, Jun. 2009, pp. 2674-2678.

[28] S. Sharifi, A. K. Tanc, and T. M. Duman, "LDPC code design for the two-user Gaussian multiple access channel," IEEE Trans. Wireless Commun., vol. 15, no. 4, pp. 2833-2844, Apr. 2016.

[29] A. Balatsoukas-Stimming, S. Rini, and J. Kliewer, "LDPC coded multiuser shaping for the Gaussian multiple access channel," in Proc. IEEE Int. Symp. Inf. Theory (ISIT), Jul. 2019, pp. 2609-2613.

[30] S. Boyd and L. Vandenberghe, Convex optimization. New York, NY, USA: Cambridge Univ. Press, 2004.

[31] E. Casini, R. D. Gaudenzi, and O. D. R. Herrero, "Contention resolution diversity slotted ALOHA (CRDSA): An enhanced random access scheme for satellite access packet networks," IEEE Trans. Wireless Commun., vol. 6, no. 4, pp. 1408-1419, Apr. 2007.

[32] E. Paolini, G. Liva, and M. Chiani, "Coded slotted ALOHA: A graphbased method for uncoordinated multiple access," IEEE Trans. Inf. Theory, vol. 61, no. 12, pp. 6815-6832, Dec. 2015.

[33] E. Biglieri and M. Lops, "Multiuser detection in a dynamic environment-part I: User identification and data detection," IEEE Trans. Inf. Theory, vol. 53, no. 9, pp. 3158-3170, Sep. 2007.

[34] J. G. Andrews, F. Baccelli, and R. K. Ganti, "A tractable approach to coverage and rate in cellular networks," IEEE Trans. Commun., vol. 59, no. 11, pp. 3122-3134, Nov. 2011.

[35] IEEE Standard For Information Technology_Telecommunications And Information Exchange Between Systems-Local And Metropolitan Area Networks-Specific Requirements-Part 11: Wireless Lan Medium Access Control (Mac) And Physical Layer (Phy) SpecificationsAmendment 4: Enhancements For Very High Throughput For Operation In Bands Below $6 \mathrm{GHz}$, IEEE Standards 802.11ac(TM)-2013, Dec. 2013, pp. 1-425.

[36] R. Jain, D.-M. Chiu, and W. R. Hawe, A Quantitative Measure of Fairness and Discrimination for Resource Allocation in Shared Computer System. Eastern Research Laboratory, Digital Equipment Corporation, Hudson, MA, USA, 1984, vol. 38.

[37] G. Liva, "Graph-based analysis and optimization of contention resolution diversity slotted ALOHA," IEEE Trans. Commun., vol. 59, no. 2, pp. 477-487, Feb. 2011.

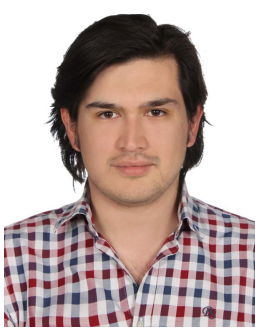

Nurullah Karakoc (Student Member, IEEE) received the B.Sc. and M.Sc. degrees in electrical and electronics engineering from Bilkent University, Turkey, in 2015 and 2017, respectively. He is currently pursuing the Ph.D. degree with Arizona State University, Tempe, AZ, USA. His current research interests include wireless communications, networking, and optimization theory.

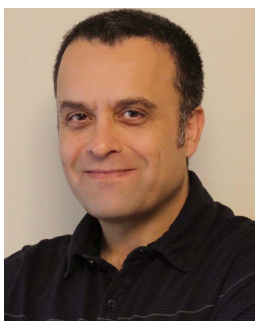

Tolga M. Duman (Fellow, IEEE) received the B.S degree from Bilkent University, Ankara, Turkey, in 1993, and the M.S. and Ph.D. degrees from Northeastern University, Boston, MA, USA, in 1995 and 1998 , respectively, all in electrical engineering. Prior to joining Bilkent University in September 2012, he was with the School of ECEE, Arizona State University, as an Assistant Professor from 1998 to 2004, an Associate Professor from 2004 to 2008, and a Professor after 2008. He is currently a Professor with the Electrical and Electronics Engineering Department, Bilkent University. His current research interests include systems, with particular focus on communication and signal processing, including wireless and mobile communications, coding/modulation, coding for wireless communications, data storage systems, and underwater acoustic communications. He was a recipient of the National Science Foundation CAREER Award and the IEEE Third Millennium Medal. 